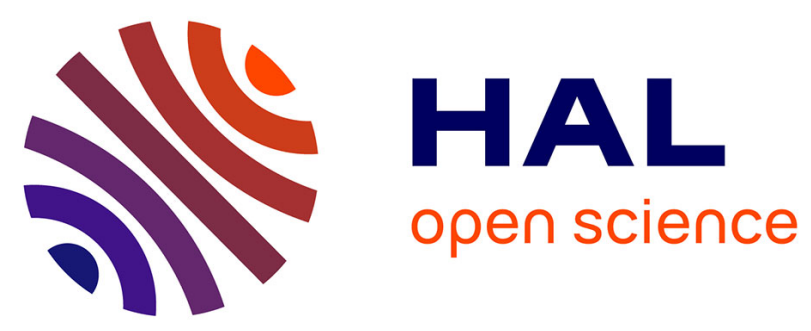

\title{
How the Size and Density of Charge-Transfer Excitons Depend on Heterojunction's Architecture
}

Marcio T Do N. Varella, Ljiljana Stojanović, van Quan Vuong, Stephan Irle, Thomas A Niehaus, Mario Barbatti

\section{- To cite this version:}

Marcio T Do N. Varella, Ljiljana Stojanović, van Quan Vuong, Stephan Irle, Thomas A Niehaus, et al.. How the Size and Density of Charge-Transfer Excitons Depend on Heterojunction's Architecture. Journal of Physical Chemistry C, 2021, 125 (10), pp.5458-5474. 10.1021/acs.jpcc.0c10762 . hal03158586

\section{HAL Id: hal-03158586 \\ https://hal.science/hal-03158586}

Submitted on 4 Mar 2021

HAL is a multi-disciplinary open access archive for the deposit and dissemination of scientific research documents, whether they are published or not. The documents may come from teaching and research institutions in France or abroad, or from public or private research centers.
L'archive ouverte pluridisciplinaire HAL, est destinée au dépôt et à la diffusion de documents scientifiques de niveau recherche, publiés ou non, émanant des établissements d'enseignement et de recherche français ou étrangers, des laboratoires publics ou privés. 


\title{
How size and density of charge transfer excitons depend on heterojunction's architecture
}

\author{
Márcio T. do N. Varella, ${ }^{1, a)}$ Ljiljana Stojanović, ${ }^{2}$ Van Quan Vuong, ${ }^{3}$ Stephan Irle, ${ }^{3,4}$ \\ Thomas A. Niehaus, ${ }^{5}$ and Mario Barbatti ${ }^{6}$ \\ 1) Instituto de Física, Universidade de São Paulo, Rua do Matão 1371, 05508-090, \\ São Paulo, São Paulo, Brazil \\ ${ }^{2}$ School of Biological and Chemical Sciences, Queen Mary University of London, \\ Mile End Road, London, E1 $4 N S, U K$ \\ 3) Bredesen Center for Interdisciplinary Research and Graduate Education, \\ University of Tennessee, Knoxville, TN 37996, United States \\ 4) Computational Sciences and Engineering Division, Oak Ridge National Laboratory, \\ Oak Ridge, TN 37831, United States \\ 5) Univ Lyon, Université Claude Bernard Lyon 1, CNRS, Institut Lumière Matière, \\ F-69622 Lyon, France \\ 6) Aix Marseille University, CNRS, ICR, Marseille, France
}

${ }^{0}$ NOTICE OF COPYRIGHT: This manuscript has been co-authored by UT-Battelle, LLC under Contract No. DE-AC05-00OR22725 with the U.S. Department of Energy. The United States Government retains and the publisher, by accepting the article for publication, acknowledges that the United States Government retains a non-exclusive, paid-up, irrevocable, worldwide license to publish or reproduce the published form of this manuscript, or allow others to do so, for United States Government purposes. The Department of Energy will provide public access to these results of federally sponsored research in accordance with the DOE Public Access Plan (http://energy.gov/downloads/doe-public-access-plan). 
We have characterized the size, intensity, density, and distribution of charge transfer (CT) excitons as a function of the acceptor-donor architecture of prototypical organic interfaces. This characterization was done by computational analysis of 17 interface models, changing number, position, and orientation of the donor and acceptor molecules. The interfaces' building blocks were PCBM fullerene acceptors and dual-band donor polymers composed of thiophene, benzothiadiazole, and benzotriazole subunits. The interfaces' electronic structure was computed with the time-dependent long-range-corrected density-functional based tight-binding method and analyzed with the fragment-based one-electron transition density matrix. In all models, the interfaces with edge-on orientation have denser spectra of low-energy CT states lying below the absorption bands compared to the interfaces with face-on orientation. This CT-state distribution in edge-on interfaces provides a gate to efficiently populate cold CT excitons. Moreover, the cold CT excitons have a higher degree of charge separation in the edge-on than in the face-on interfaces. The CT amount and the CT exciton size generally increase with the energy of the CT states, although the electron remains localized on a single molecule in cold CT states. Delocalization over two PCBM molecules was observed for high-energy CT states. The exciton size also depends on the orientation. Larger excitons are produced by the delocalization of the electron perpendicularly to the interface. When the delocalization is parallel, the smaller electron-hole distances yields moderately sized CT excitons.

a)Electronic mail: mvarella@if.usp.br 


\section{INTRODUCTION}

Efficient charge separation at donor-acceptor (DA) interfaces is critical for the performance of electronic devices. In organic solar cells (OSCs), light absorption typically gives rise to bound excitons, and their dissociation into charge-separated (CS) states can be significantly constrained by radiative and non-radiative recombination processes ${ }^{1,2}$. Chargetransfer (CT) excitons at the interface between donor and acceptor domains attracted considerable attention in recent years ${ }^{3-7}$, since they are believed to mediate the long-range charge separation in OCSs. Despite the outstanding progress in conversion efficiency ${ }^{8-11}$, the mechanisms for free-carrier production are still under debate. For instance, the generation of free charges from high-energy (hot) CT excitons has been pointed out ${ }^{12-14}$, but significant yields have also been reported for light absorption at sub-excitation energies ${ }^{15,16}$. The dissociation mechanisms of interfacial CT excitons, having binding energies 10 times higher than ambient thermal energies ${ }^{1,15}$, are also currently in discussion. Several physical effects can impact the exciton dissociation dynamics, such as entropy ${ }^{6,17-19}$, vibronic couplings ${ }^{19-22}$, energy offset ${ }^{5,22-31}$, disorder ${ }^{32-36}$, hybridization between CT and localized $\operatorname{states}^{7,22,37-39}$, and also electronic delocalization and polarization ${ }^{14,34,40-50}$, among others.

A number of recent studies have addressed the microscopic structures of the DA interfaces and their impact on free-carrier-generation ${ }^{5-7,34,38,46,47,51-54}$. While atomistic simulations can provide invaluable mechanistic and structure-property insights, the importance of going beyond minimal (bimolecular) interface models has been emphasized ${ }^{34,47,52,54}$. Therefore, the computational methods are challenged by the size of the DA heterojunction models, by the need to account for different interface geometries, and also by disorder and finitetemperature effects. The balance between numerical effort and accuracy is a prominent issue, but the analysis of the excited states of complex molecular systems should not be overlooked. The inspection of molecular orbitals is often tedious and sometimes ineffective to assign the CT character of electronic states, e.g., when many electron-hole pairs have significant weights in a given excited state or for systems having multiple chromophores.

In this work, we address the CT excitons of polymer:fullerene interfaces combining the time-dependent long-range corrected density functional tight-binding (TD-LC-DFTB) method $^{56-58}$ with the fragment-based analysis of the calculated excited-states ${ }^{59-61}$. The fragmentation into subsystems has proved a useful tool to assign, quantitatively and un- 
ambiguously, the CT character of exciton states. Its application to DA heterojunctions is particularly appealing since the interface can be viewed as composed of donor and acceptor fragments, which can be further decomposed into oligomer and fullerene fragments. The methodology is applied to an interface model in which the acceptor domain is composed of PCBM (phenyl-C61-butyric acid methyl ester), and the donor domain by PTBTBTz, a copolymer comprised of thiophene $(\mathrm{T})$, benzothiadiazole $(\mathrm{BT})$, and benzotriazole $(\mathrm{BTz})$ subunits, as shown in Fig. 1a. PTBTBTz is an example of D-A $\mathrm{A}_{1}-\mathrm{D}-\mathrm{A}_{2}$ dual-band polymers ${ }^{62}$, which have been considered for applications in photovoltaics ${ }^{62-65}$ and organic electronics ${ }^{66,67}$. Those copolymers are characterized by bright excitations from the donor subunit ( $\mathrm{T}$ in the present case) to the strong and weak acceptor subunits (BT and BTz) - the latter acceptor subunits in the PTBTBTz donor domain should not be confused with the acceptor domain of the interface model.

The PTBTBTz:PCBM interface is an interesting model for our purposes since (i) in addition to cold and hot CT excitons, the dual-band character is expected to give rise to interband CT states; (ii) the relation between interface geometry and CT states can be affected by the two absorption bands arising from electron transfer to two distinct polymer subunits; and (iii) the BT and BTz acceptor subunits are moderately sized compared to those employed in other $\mathrm{D}-\mathrm{A}_{1}-\mathrm{D}-\mathrm{A}_{2}$ polymers $^{62}$, thus helping to manage the computational effort. We explore 17 DA interface models of different sizes and geometries obtained from both geometry optimizations and room-temperature molecular dynamics (MD) simulations. The excited state analysis employed different fragmentation schemes and exciton properties to draw, as much as possible, general conclusions not restricted to the properties of the PTBTBTz:PCBM system. We explore edge-on and face-on orientations of the DA domains, which are known to correlate to device performance $6,47,51,52$, different closest-approach positions of the acceptor molecules along the donor chains, and also the size of the interface domais. The fragment-based analysis is further applied to physical properties expected to affect the excited state dynamics, such as the degree of charge transfer, delocalization of the CT excitons, and the electron-hole distances. 


\section{METHODS}

The excitation spectra were calculated with the TD-LC-DFTB method, described in detail elsewhere $\mathrm{f}^{57,58}$, as implemented in the DFTB $+\operatorname{code}^{69}$. From the formal standpoint, the approach is similar to linear-response time-dependent density functional theory (TDDFT), i.e., one has to solve the generalized eigenvalue equation

$$
\left[\begin{array}{cc}
\mathrm{A} & \mathrm{B} \\
\mathrm{B}^{*} & \mathbf{A}^{*}
\end{array}\right]\left[\begin{array}{l}
\mathrm{X} \\
\mathrm{Y}
\end{array}\right]=\Omega\left[\begin{array}{cc}
1 & 0 \\
0 & -1
\end{array}\right]\left[\begin{array}{l}
\mathrm{X} \\
\mathrm{Y}
\end{array}\right]
$$

where the $\mathbf{A}$ and $\mathbf{B}$ matrices are given by

$$
\begin{aligned}
& A_{i a \sigma, j b \sigma^{\prime}}=\delta_{i j} \delta_{a b} \delta_{\sigma \sigma^{\prime}}\left(\varepsilon_{a \sigma}-\varepsilon_{i \sigma}\right)+\left(i a \sigma \| j b \sigma^{\prime}\right) \\
& B_{i a \sigma, j b \sigma^{\prime}}=\left(i a \sigma \| b j \sigma^{\prime}\right)
\end{aligned}
$$

In the expressions above, $\Omega$ denotes the excitation energies (eigenvalues), $\varepsilon_{i \sigma, a \sigma}$ are orbital energies obtained from a previous ground-state calculation for spin $\sigma$, and $\left(i a \sigma \| j a \sigma^{\prime}\right)$ are generalized electron repulsion matrix elements, which involve occupied $(i, j)$ and virtual $(a, b)$ orbitals, and account for the Hartree and exchange-correlation interactions. In TDDFTB the two-electron integrals are computed with a Mulliken approximation to increase the numerical efficiency ${ }^{55}$.

The TD-LC-DFTB method is built on the the LC-DFTB counterpart. The Coulomb interaction is split into short- and long-range components using a Yukawa ansatz ${ }^{57}$, and the DFTB approximation for the short-range contribution is obtained from the Baer, Neuhauser, and Livshits (BNL) exchange-correlation functional ${ }^{71,72}$. A second-order expansion of the Kohn-Sham total energy around a reference density matrix is carried out, $\rho=\rho_{0}+\delta \rho$, where $\rho_{0}$ is the sum of BNL atomic density matrices. While we do not discuss the approximations employed to obtain the DFTB ground state energies ${ }^{70}$, we mention the decomposition of the total energy into two contributions, $E_{\text {total }}=E_{\text {el }}+E_{\text {rep }}$. The electronic part, $E_{\text {el }}$, involves the computation of the Hamiltonian matrix elements which only depend on the reference density. The corresponding diagonal elements are given by the atomic orbital energies, while the off-diagonal elements are precomputed for pairs of elements over a range of internuclear distances, along with the overlap matrix and the repulsive energy component. These precomputed values are tabulated in the Slater-Koster (SK) files ${ }^{70}$. The electronic part further 
accounts for deviations from the reference density through a charge-charge interaction term.

An SK parametrization with the range-separation parameter $\omega=0.3 a_{0}^{-1}$, referred to as the OB2 set, was recently reported for the H, C, N, and O elements ${ }^{73}$. The present calculations used a re-parametrization of this OB2 set, which includes sulfur and was optimized for five different values of the range-separation parameter, namely $\omega=0.1,0.2,0.3,0.4$, and $0.5 a_{0}{ }^{-1}$. The parameter sets used in this work are deposited in the Supporting Information. The inclusion of sulfur is essential for applications in organic electronics, and the range of $\omega$ values allows for a partial tuning of range separation, which is considered important to describe CT excitations ${ }^{4}$. The complete re-parameterization of the OB2 set will be published elsewhere in the near future.

Our LC-DFTB and TD-LC-DFTB calculations included dispersion interactions via the Slater-Kirkwood model ${ }^{74}$. The ground-state MD simulations were based on LC-DFTB energies and gradients either in the NVE or NVT ensemble. In the latter case, the Andersen thermostat, built in the DFTB + code, was employed, with a reselection probability of 0.2. We did not account for the dielectric environment since continuum solvation models are currently not available in the DFTB + package. A similar study of polymer:fullerene (P3HT:PCBM) interfaces ${ }^{52}$ pointed out that accounting for the dielectric medium essentially gives rise to a systematic shift of the excitation spectra, not significantly affecting the photophysics. We expect that the lack of a solvation model should not affect the main conclusions of the present work, as they rely only on the relative energies of the excited states. Finally, the charge separation parameter was set to $\omega=0.2 a_{0}^{-1}$ for the PTBTBTz:PCBM interface models. This value was chosen because it provided, among the available $\omega$ values, the smallest $\left|\mathrm{IP}-\left(E^{+}-E^{0}\right)\right|$ differences (in absolute value), where $E^{+}$and $E^{0}$ are the energies of the cationic and neutral species, and IP is the ionization potential of the latter.

Other supramolecular models of interest for organic electronics have been recently investigated using an alternative version of TD-LC-DFTB ${ }^{56,81-83}$. In addition to a different parametrization, that version also differs from the present one by not including Hartree-Fock exchange in the DFTB zeroth-order Hamiltonian (see Ref. 58). In a nonadiabatic dynamics study of a pentacene:fullerene interface based on that TD-LC-DFTB version, Darghouth and co-workers ${ }^{83}$ have proposed a value for $\omega, 0.07 a_{0}^{-1}$, significantly smaller than those used in our simulations.

The exciton analysis employed the recently developed ${ }^{75}$ interface between the DFTB $+{ }^{69}$ 
and TheoDORE ${ }^{76}$ codes. The latter is a toolbox for the fragment-based analysis of excited states $^{61}$, exploring the one-electron transition density matrix (1-TDM) between the ground and the $I$-th excited state, $\gamma^{0 I}\left(\mathbf{r}_{e}, \mathbf{r}_{h}\right)$, where the electron and hole coordinates are indicated as $\mathbf{r}_{e, h}$. Groups of atoms belonging to the system of interest define molecular fragments. A charge transfer number, for fragments $A$ and $B$, can be obtained from the 1TDM by restricting the integrations over the electron and hole coordinates to the $A$ and $B$ fragments, respectively,

$$
\Omega_{A B}=\int_{A} d \mathbf{r}_{h} \int_{B} d \mathbf{r}_{e} \gamma_{0 I}^{2}\left(\mathbf{r}_{e}, \mathbf{r}_{h}\right)
$$

$\Omega_{A B}$ gives the probability to find the hole on fragment $A$, with the electron in fragment $B$, and it can be evaluated by resorting to a population analysis procedure ${ }^{59,61}$. The interpretation of $\Omega_{A B}$ as a matrix defines the electron-hole correlation plots and allows for the definition of several excited-state descriptors ${ }^{59}$. The CT number is obtained from the off-diagonal elements,

$$
\mathrm{CT}=\frac{1}{\Omega} \sum_{A} \sum_{B \neq A} \Omega_{A B}
$$

with $\Omega=\sum_{A, B} \Omega_{A B}$, and it ranges from $\mathrm{CT}=0$ (ideal Frenkel exciton) to $\mathrm{CT}=1$ (ideal charge-transfer exciton). In case the fragments can be arranged along some direction, such that an ordering like $A=1, B=2$, etc. can be meaningfully assigned, the position descriptor (POS) for the hole $(h)$ and electron $(e)$ orbitals can be computed as,

$$
\begin{aligned}
& \operatorname{POS}_{h}=\Omega^{-1} \sum_{A} A\left(\sum_{B} \Omega_{A B}\right) \\
& \operatorname{POS}_{e}=\Omega^{-1} \sum_{B} B\left(\sum_{A} \Omega_{A B}\right)
\end{aligned},
$$

with the exciton position given by

$$
\mathrm{POS}=\frac{1}{2}\left(\mathrm{POS}_{h}+\mathrm{POS}_{e}\right) .
$$

Ideal Frenkel excitons localized on the donor (LD) and on the acceptor (LA) are represented in Fig. 1b, along with an ideal CT exciton. The CT and POS descriptors for these excited states are also indicated, and the corresponding electron-hole correlation plots are shown in Fig. 1c. The POS descriptor is calculated with the convention $\mathrm{D}=1$ and $\mathrm{A}=2$ for the 
positions of the donor and acceptor, respectively, whenever the system is decomposed into two fragments.

The 1TDM can also be interpreted as the exciton wave function ${ }^{60}, \chi_{\operatorname{exc}}\left(\mathbf{r}_{e}, \mathbf{r}_{h}\right):=$ $\gamma^{0 I}\left(\mathbf{r}_{e}, \mathbf{r}_{h}\right)$, which allows for the definition of the exciton size as the root-mean-square (rms) distance between the hole and the electron,

$$
d_{\mathrm{exc}}^{2}=\frac{\left\langle\chi_{\mathrm{exc}}\left|\left(\mathbf{r}_{h}-\mathbf{r}_{e}\right)^{2}\right| \chi_{\mathrm{exc}}\right\rangle}{\left\langle\chi_{\mathrm{exc}} \mid \chi_{\mathrm{exc}}\right\rangle}
$$

The rms distance can be approximately evaluated by defining atomic pairs, breaking the integrals into atomic volumes, and assuming the electron-hole distance to be equal to the distance between the nuclei $\left(d_{M N}\right)$ for each atomic pair ${ }^{60}$,

$$
d_{\mathrm{exc}} \approx \sqrt{\frac{1}{\Omega} \sum_{M, N} \Omega_{M N} d_{M N}^{2}},
$$

where $\Omega_{M N}$ is the $\Omega$ matrix, given in eq. 3, for the single-atom fragments $M$ and $N$.

Fig. 1(d) shows the CT and POS descriptors for the 50 lowest-lying singlet excited states of 10 interface models out of those considered in the present study. The systems were decomposed into two fragments, corresponding to the donor (D) and acceptor (A) domains, and the POS descriptors were calculated with the positions of the D and A fragments chosen as 1 and 2, respectively (see eqs. 5 and 6). The data points are somewhat scattered, making the assignment of Frenkel and CT excitons arbitrary to some extent. Nevertheless, most of the points lie close to the corners of a triangle whose vertices correspond to the ideal exciton characters, namely LD $(\mathrm{CT}=0.0, \mathrm{POS}=1.0), \mathrm{LA}(\mathrm{CT}=0.0, \mathrm{POS}=2.0)$, and CT $(\mathrm{CT}=1.0, \mathrm{POS}=1.5)$. We, therefore, employ the following practical definitions,

$$
\begin{aligned}
& \mathrm{LD} \text { exciton }=\left\{\begin{array}{c}
0 \leq \mathrm{CT} \leq 0.25 \\
1.00 \leq \mathrm{POS} \leq 1.25
\end{array},\right. \\
& \mathrm{LA} \text { exciton }=\left\{\begin{array}{c}
0 \leq \mathrm{CT} \leq 0.25 \\
1.75 \leq \mathrm{POS} \leq 2.00
\end{array},\right. \\
& \mathrm{CT} \text { exciton }=\left\{\begin{array}{c}
0.75 \leq \mathrm{CT} \leq 1.00 \\
1.35 \leq \mathrm{POS} \leq 1.65
\end{array},\right.
\end{aligned}
$$


and also assign those not matching the above definitions as mixed-character (MX) excitons. Although we explore different fragmentation schemes, the interfacial CT excitons are always defined using only two fragments corresponding to the D and A domains.

(a)


$\mathrm{BTz}$

(b)

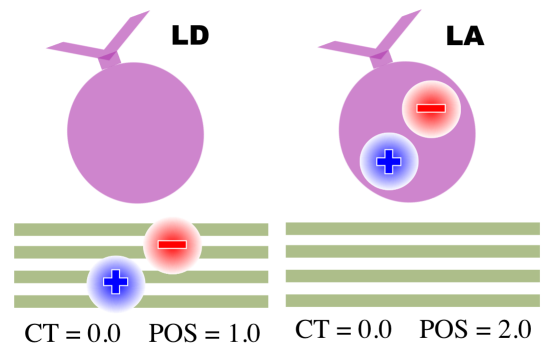

(d)

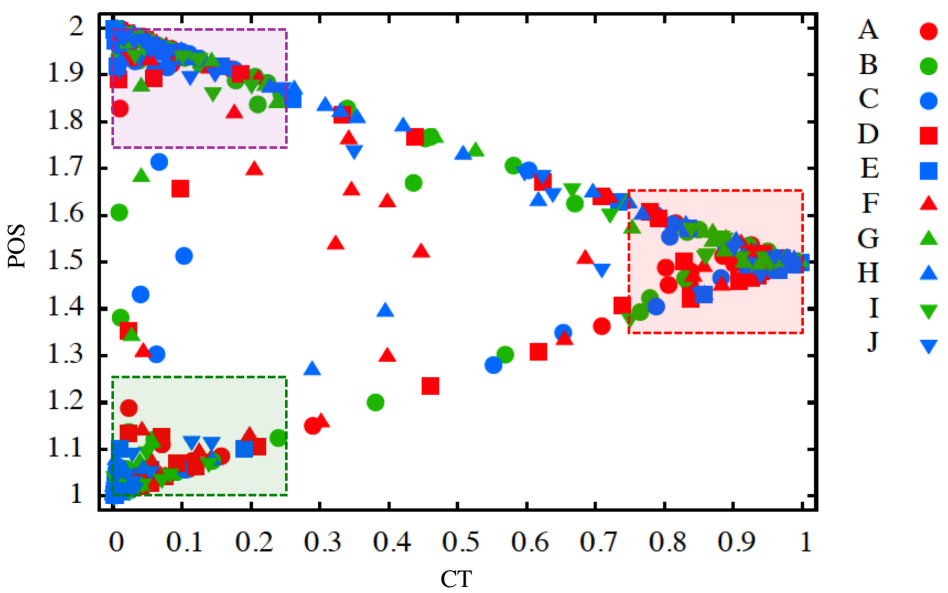

(c)
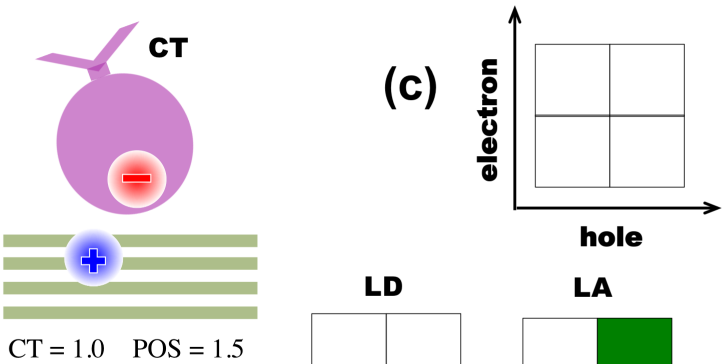
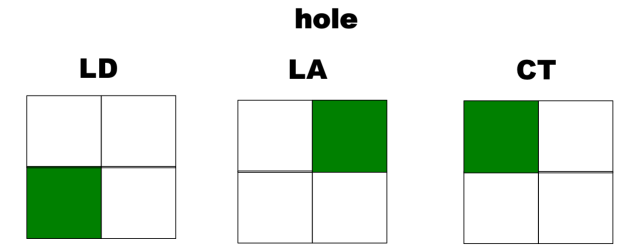

FIG. 1. (a) Structures of PCBM and PTBTBTz monomer, with the T, BT, and BTz subunits indicated for the latter. The sulfur atoms are represented in yellow, oxygen in red, nitrogen in deep blue, carbon in light blue, and hydrogen in white. (b) Ideal excited states: Frenkel exciton localized on the donor domain (LD); Frenkel exciton localized on the acceptor domain (LA); and charge-transfer exciton (CT). In the three panels, the electron and hole locations are indicated by the negative (red) and positive (blue) signs. The values of the CT and POS descriptors for the ideal excitations are also given in the panels, according to eqs. 4 and 6 . The donor and acceptor positions are defined as 1 and 2, respectively. (c) Electron-hole correlation plots for the ideal LD, LA, and CT excited states, according to eq. 3. The electron and hole axes are also shown. (d) CT and position (POS) descriptors for the excited states of several interface models, where the lowest-lying 50 excited states were calculated in each case. The model systems are $(\mathrm{A})$ $(\operatorname{dim}-\mathrm{PT})_{2}:(\mathrm{PCBM} @ b t)_{1}: f ;(\mathrm{B})(\operatorname{dim}-\mathrm{PT})_{2}:(\mathrm{PCNM} @ b t z)_{1}: f ;(\mathrm{C})(\operatorname{dim}-\mathrm{PT})_{2}:(\mathrm{PCBM} @)_{1}: f ;(\mathrm{D})$ $(\operatorname{dim}-\mathrm{PT})_{2}:(\mathrm{F} @ b t)_{1}: e ;(\mathrm{E})(\operatorname{dim}-\mathrm{PT})_{2}:(\mathrm{PCBM} @)_{1}: e ;(\mathrm{F})(\operatorname{dim}-\mathrm{PT})_{3}:(\mathrm{PCBM} @ b t)_{1}: f ;(\mathrm{G})(\operatorname{dim}-$ $\mathrm{PT})_{3}:(\mathrm{PCBM} @ b t z)_{1}: f ;(\mathrm{H})(\text { dim-PT) })_{3}:(\mathrm{PCBM} @)_{1}: f ;(\mathrm{I})(\text { dim-PT3) })_{2}:(\mathrm{PCBM} @ b t z)_{1}: f ;(\mathrm{J})($ dimPT3 $)_{2}:(\mathrm{PCBM} @)_{1}: f$. The shaded areas highlight the practical definitions of LD (green), LA (purple), and CT (red) excitons. 


\section{RESULTS}

Before we discuss the models for PTBTBTz:PCBM interfaces, we comment on the quality of the TD-LC-DFTB calculations compared to the TD-DFT counterparts. Table I presents the excitation energies and oscillator strengths for five lowest-energy excited states, $\mathrm{S}_{1}$ to $\mathrm{S}_{5}$, of the PTBTBTz monomer (Fig. 1a) and the stacked dimer, PTBTBTz 2 (the latter is shown in the Supporting Information, Fig. S1). The DFT and TD-DFT computations were performed with the Gaussian09 package 77 employing different correlation-exchange functionals, standard values for the range separation parameters, and the 6-31G* basis set. For PBTBTz and $\mathrm{PBTBTz}_{2}$, the TD-LC-DFTB range separation parameter was set to $\omega=0.3 a_{0}^{-1}$, based on the same criterion that was described in Sec. II. We first compare the excitation spectrum of the PTBTBTz monomer obtained with CAM-B3LYP, LC- $\omega$ PBE, $\omega \mathrm{B} 97 \mathrm{XD}$, as well as TD-LC-DFTB, at the ground-state geometry optimized with the B3LYP functional. There is a good agreement $(\lesssim 0.1 \mathrm{eV})$ between the CAM-B3LYP and $\omega$ B97XD excitation energies, while LC- $\omega \mathrm{PBE}$ overestimates them by $\approx 0.4 \mathrm{eV}$ with respect to the other functionals. The discrepancies between TD-LC-DFTB energies and those obtained with CAM-B3LYP and $\omega$ B97XD vary more significantly from state to state, being mostly around $\approx 0.1 \mathrm{eV}$ to $\approx 0.3 \mathrm{eV}$, although larger for the $\mathrm{S}_{3}$ state, $\approx 0.5 \mathrm{eV}$. The agreement between TD-LC-DFTB and $\omega$ B97XD is similar at the ground-state geometry optimized with the latter functional, although there is some improvement when we compare the excitation energies computed at the respective optimal geometries $(\lesssim 0.4 \mathrm{eV}$ discrepancies $)$. For the PTBTBTz $_{2}$ dimer, the discrepancies between the TD- $\omega$ B97XD and TD-LC-DFTB energies are somewhat lower, in spite of the less favorable agreement for the $\mathrm{S}_{1}$ state.

The dual-band character of the PTBTBTz polymer is already evident in the monomer. As discussed elsewhere ${ }^{62}$, the bright $S_{1}$ and $S_{2}$ states mainly arise from excitations out of the T subunit to the BT and BTz subunits, respectively. The oscillator strengths calculated with the TD-DFT and TD-LC-DFTB methods are consistent, with $f \approx 1$ and $f \approx 0.1$ for the transitions to the $S_{1}$ and $S_{2}$ states, respectively. The situation is similar for the dimer, although with larger differences in the magnitudes of the oscillator strengths, especially when TD-LC-DFTB and TD- $\omega$ B97XD are compared at their respective optimal geometries. Particularly at regions where the excited-state spectra are denser, the oscillator strengths, and even the character of the excited states, should be more strongly dependent on the 
geometry. To further inspect this aspect, we calculated the absorption spectrum of the monomer by performing a Monte Carlo integration of the Wigner distribution function of the vibrational ground state ${ }^{78}$. The simulated spectra, shown in the Supporting Information (Section S-II), were calculated with the Newton-X code $^{79,80}$ using the excitation energies, oscillator strengths, and vibrational normal modes obtained with the TD- $\omega$ B97XD and TDLC-DFTB methods. The shapes of the absorptions bands and the overall magnitudes of the cross sections are in good agreement, despite the $\approx 0.2 \mathrm{eV}$ shift between the maxima of the first bands, which could be anticipated from the vertical excitations reported in Table I.

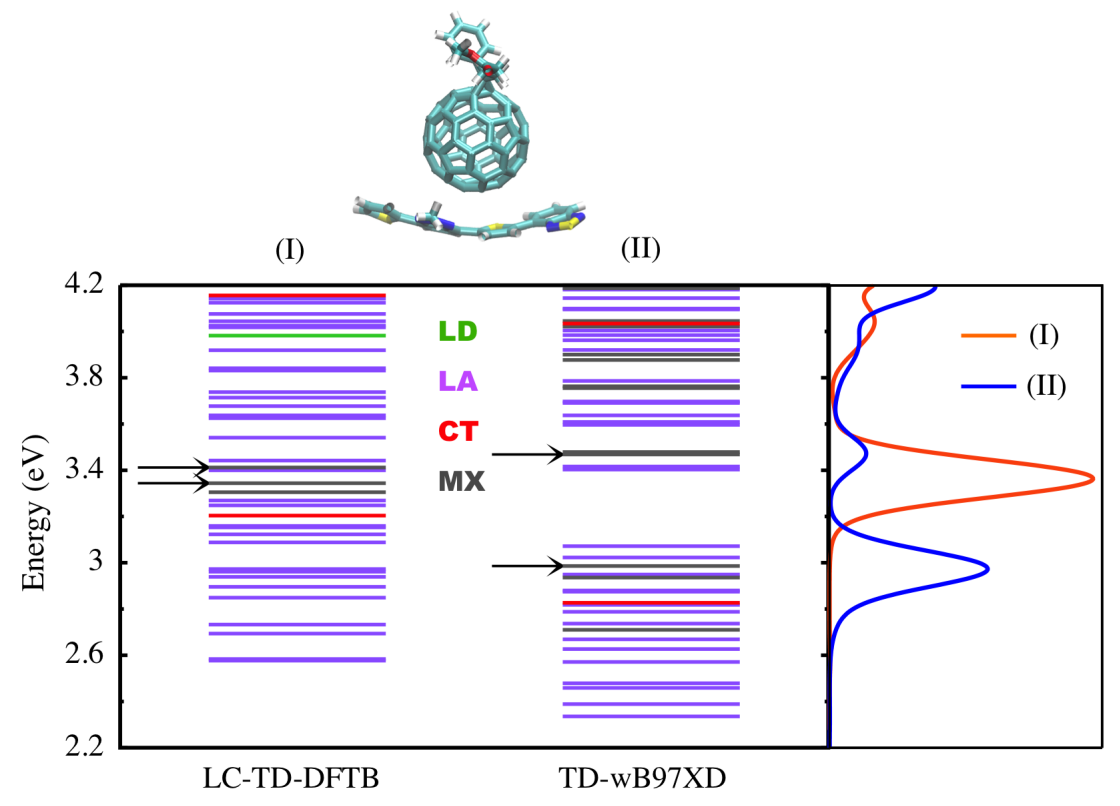

FIG. 2. The main panel shows the vertical excitation spectra for the PTBTBTz:PCBM dimer obtained with the (I) TD-LC-DFTB and (II) TD- $\omega$ B97XD/6-31G* methods at their respective ground-state stationary geometries. The LD states are indicated in green, the LA in purple, the $\mathrm{CT}$ in red, and MX in gray. The horizontal arrows indicate the low-energy bright transitions. The right panel shows the absorption cross section (in arbitrary units) obtained from the broadening of the vertical spectra with Gaussian line shapes.

The excited states for a minimal PBTBTz:PCBM interface model were computed with the TD-LC-DFTB and TD- $\omega$ B97XD/6-31G* methods at the ground state geometries optimized with LC-DFTB and DFT- $\omega \mathrm{B} 97 \mathrm{XD} / 6-31 \mathrm{G}^{*}$, respectively. The range-separation parameters were tuned, in the TD calculations, to $\omega=0.3$ (TD-LC-DFTB) and $\omega=0.14$ (TD$\left.\omega \mathrm{B} 97 \mathrm{XD} / 6-31 \mathrm{G}^{*}\right)$. The results are shown in Fig. 2, where the LD, LA, CT, and MX excitons defined in eqs. 9 to 11 are indicated, along with approximate absorption cross sections, in arbitrary units. The latter correspond to the vertical spectra broadened with Gaussian profiles 
having a full width at half maxima of $0.1 \mathrm{eV}$ and vertical oscillator strengths corresponding to their area. In general, the TD-LC-DFTB spectrum is blue-shifted by $\approx 0.3 \mathrm{eV}$, and the two lowest-lying absorption bands obtained from the TD-DFTB/ $\omega$ B97XD/6-31G* calculation, at $\approx 3.0 \mathrm{eV}$ and $\approx 3.4 \mathrm{eV}$, merge into a single stronger band in the TD-LC-DFTB results. However, the photophysics expected from both models would be similar since the bottom of the spectra has high densities of LA states with a single CT state lying $\approx 0.2 \mathrm{eV}$ below the brightest states. The comparison between TD-LC-DFTB and the TD- $\omega$ B97XD/6-31G* model, largely employed in atomistic models for OSCs, is encouraging. While the TD-LCDFTB excitation energies are overestimated, the relative energies between the LD, LA, and CT should be more relevant to the excited state dynamics. Several features of the present results are consistent with TD-DFT computations for DA interfaces ${ }^{46,47,52}$, as discussed below. The efficiency of the TD-LC-DFTB method is important since we consider about 20 systems with 252 to 412 atoms, exploring $\approx 110$ geometries obtained from MD simulations.

The structures of the interface models, with varying compositions and conformations, are presented in Fig. 3 and in the Supporting Information, Figs. S2 and S3. Their Cartesian coordinates are also given in the Supporting information. The donor domains are formed by stacked PTBTBTz oligomers in most cases, although we have performed a few calculations with PT3BTBTz, which has also been considered for $\mathrm{OSCs}^{62}$. In the latter, the BT and BTz subunits are intercalated by three T subunits, as shown in the Supporting Information, Fig. S1. A donor formed by $n$ stacked chains of PTBTBTz or PT3BTBTz oligomers is denoted as $\left(n_{\text {mon }}-\mathrm{PT}\right)_{n}$ or $\left(n_{\text {mon }}-\mathrm{PT} 3\right)_{n}$, respectively, where $n_{\text {mon }}=\operatorname{dim}$, tri, and tet, respectively for dimers, trimers, and tetramers, while $n=2,3$. The acceptor domains are composed of either one or two PCBM molecules, and we consider their position and orientation with respect to the donor. For a single PCBM molecule, the position is defined by the closest-lying donor subunit (T, BT or BTz), while the orientations are face-on $(f)$ or edge-on $(e)$ with respect to the stacked oligomers. The interface models are thus denoted as $\left(n_{\text {mon }}-\mathrm{PT}\right)_{n}(\mathrm{PCBM} @ p)_{1}: o$, where $p=\mathrm{bt}$, btz, t indicates the position (donor subunit lying the closest to the PCBM molecule), while $o=f$, e indicates the orientation. In case two PCBM subunits are present, they may be arranged side by side (2sd) or in a row (2rw). The models are therefore denoted as $\left(n_{\text {mon }}-\mathrm{PT}\right)_{n}(\mathrm{PCBM} @ p)_{2 \mathrm{rw}}: o$ and $\left(n_{\mathrm{mon}}-\mathrm{PT}\right)_{n}\left(\mathrm{PCBM} @ p_{1-} p_{2}\right)_{2 \mathrm{sd}}: o$, with $p_{1} p_{2}=\mathrm{t} \_\mathrm{t}$, bt_btz.

The excited states of interface models formed by $n=2$ stacked PTBTBTz dimers and a single PCBM molecule with face-on orientation are shown in Fig. 4(a), where the colors 


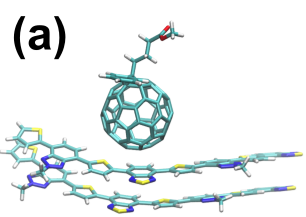

$(\operatorname{dim}-\mathrm{PT})_{2}(\mathrm{PCBM} @ \mathrm{bt})_{1}: f$

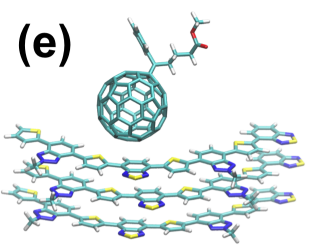

$(\text { dim-PT) })_{3}(\mathrm{PCBM} @ \mathrm{bt})_{1}: f$

(i)

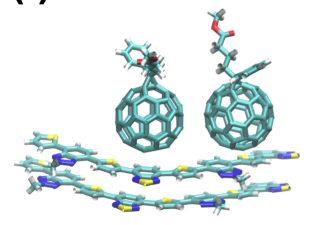

$(\text { dim-PT })_{2}(\text { PCBM@bt_btz) })_{2 s d}: f$

(m)

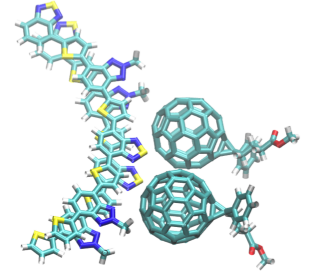

$(\operatorname{dim}-\mathrm{PT})_{2}\left(\mathrm{PCBM} @ \mathrm{t} \_\mathrm{t}\right)_{2 \mathrm{sd}}: e$

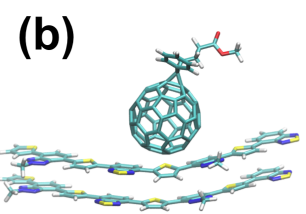

$(\operatorname{dim}-\mathrm{PT})_{2}(\mathrm{PCBM} @ \mathrm{t})_{1}: f$

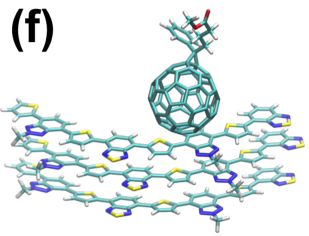

$(\operatorname{dim}-\mathrm{PT})_{3}(\mathrm{PCBM} @ \mathrm{t})_{1}: f$

(j)

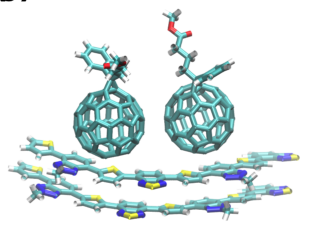

$(\operatorname{dim}-\mathrm{PT})_{2}\left(\mathrm{PCBM} @ \mathrm{t} \_\mathrm{t}\right)_{2 \mathrm{sd}}: f$

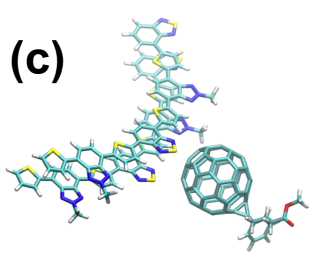

$(\operatorname{dim}-\mathrm{PT})_{2}(\mathrm{PCBM} @ \mathrm{bt})_{1}: e$

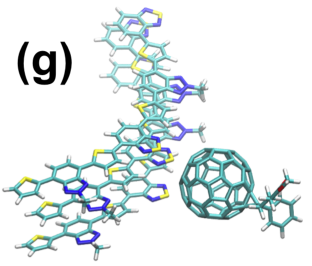

$(\mathrm{dim}-\mathrm{PT})_{3}(\mathrm{PCBM} @ \mathrm{bt})_{1}: e$

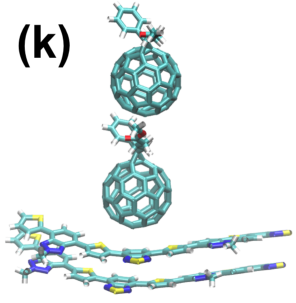

$(\operatorname{dim}-\mathrm{PT})_{2}(\mathrm{PCBM} @ \mathrm{bt})_{2 \mathrm{rw}}: f$

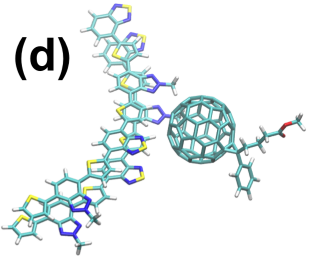

$(\operatorname{dim}-\mathrm{PT})_{2}(\mathrm{PCBM} @ \mathrm{t})_{1}: e$

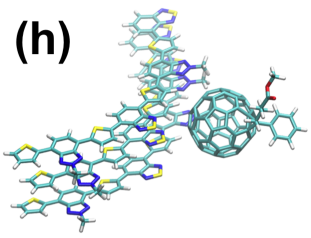

$(\mathrm{dim}-\mathrm{PT})_{3}(\mathrm{PCBM} @ \mathrm{t})_{1}: e$

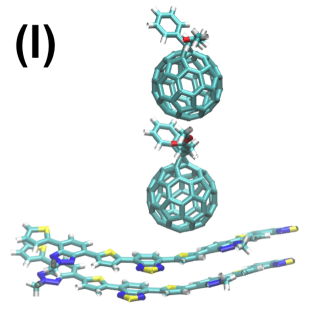

$(\operatorname{dim}-\mathrm{PT})_{2}(\mathrm{PCBM} @ \mathrm{t})_{2 \mathrm{rw}}: f$ (n)

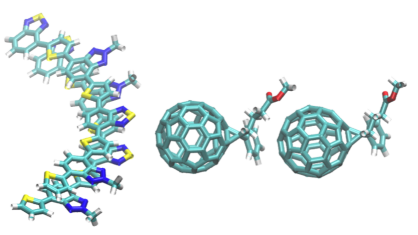

$(\operatorname{dim}-\mathrm{PT})_{2}(\mathrm{PCBM} @ \mathrm{bt})_{2 \mathrm{rw}}: e$
(0)



FIG. 3. (a) Structures of the interface models addressed in the present study (the remaining structures are shown in the Supporting Information, Figs. S2 and S3). In all panels, the donor domain is composed of stacked PTBTBTz dimers, $(\text { dim-PT })_{n}$, with $n=3$ stacked chains in panels (e) to (h), and $n=2$ stacked chains in the other panels. A single PCBM molecule forms the acceptor domain in panels (a) to (h). Its relative position, defined by the closest-lying donor subunit, is indicated as $(\mathrm{PCBM} @ p)_{1}$, where $p=\mathrm{t}$ (thiophene) in panels $(\mathrm{b}),(\mathrm{d}),(\mathrm{f}),(\mathrm{h})$, and $p=\mathrm{bt}$ (benzothiodiazole) in panels (a), (c), (e) and (g). The orientation of the PCBM subunits is either face-on, $(\mathrm{PCBM} @ p)_{1}: f$, as in panels $(\mathrm{a}),(\mathrm{b}),(\mathrm{e}),(\mathrm{f})$, or edge-on, $(\mathrm{PCBM} @ p)_{1}: e$, as in panels (c), (d), (g), (h). In panels (i) to (o), the acceptor domain is formed by two PCBM molecules, either arranged side by side $(2 \mathrm{sd})$, as in panels $(\mathrm{i}),(\mathrm{j}),(\mathrm{m})$, or in a row $(2 \mathrm{rw})$, as in panels $(\mathrm{k}),(\mathrm{l})$, $(\mathrm{n}),(\mathrm{o})$. The positions are denoted as (PCBM@ $\left.p_{1-} p_{2}\right)$, with $p_{1-} p_{2}=\mathrm{t} \_\mathrm{t}$, panels $(\mathrm{j})$ and $(\mathrm{m})$, and $p_{1} p_{2} \mathrm{~b}=\mathrm{bt} \_\mathrm{btz}$, panel (i), where btz indicates the benzotriazole donor subunit.

indicate the LD, LA, CT, and MX excitons defined in eqs. 9 to 11. The excitation spectra were obtained from stationary structures, as described in the Supporting Information (Sec- 
TABLE I. Lowest-lying excited states of the PTBTBTz monomer and the PTBTBTz 2 stacked dimer. For the monomer, we consider the five lowest-lying excited states, $\mathrm{S}_{1}$ to $\mathrm{S}_{5}$, while for dimer, $\mathrm{S}_{1}$ and the four lowest-lying bright states, $S_{\mathrm{B} 1}$ to $S_{\mathrm{B} 4}$. The energies, $E$ (in eV), and the oscillator strengths, $f$, were obtained with several methods. TD-M1//M2 indicates that the excited states were calculated with the time-dependent method M1 at the ground-state geometry optimized with the method M2. All DFT and TD-DFT calculations were performed with the $6-31 \mathrm{G}^{*}$ basis set.

\begin{tabular}{|c|c|c|c|c|c|c|c|}
\hline System & Method & & $\overline{\mathbf{S}_{1}}$ & $\overline{\overline{\mathbf{S}_{2}}}$ & $\overline{\overline{\mathbf{S}_{3}}}$ & 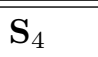 & 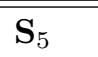 \\
\hline \multirow{14}{*}{ PTBTBTz } & TD-CAMB3LYP// & $E$ & 3.017 & 3.596 & 4.308 & 4.530 & 4.579 \\
\hline & B3LYP & $f$ & 1.022 & 0.227 & 0.023 & 0.015 & 0.036 \\
\hline & TD-LCwPBE// & $E$ & 3.378 & 4.011 & 4.740 & 4.878 & 4.993 \\
\hline & B3LYP & $f$ & 1.219 & 0.103 & 0.136 & 0.095 & 0.007 \\
\hline & TD- $\omega \mathrm{B} 97 \mathrm{XD} / /$ & $E$ & 2.917 & 3.531 & 4.382 & 4.465 & 4.530 \\
\hline & B3LYP & $f$ & 1.121 & 0.172 & 0.038 & 0.009 & 0.048 \\
\hline & TD-LC-DFTB// & $E$ & 2.997 & 3.639 & 3.842 & 4.192 & 4.201 \\
\hline & B3LYP & $f$ & 1.254 & 0.200 & 0.000 & 0.119 & 0.000 \\
\hline & TD- $\omega \mathrm{B} 97 \mathrm{XD} / /$ & $E$ & 3.097 & 3.670 & 4.538 & 4.583 & 4.651 \\
\hline & $\omega \mathrm{B} 97 \mathrm{XD}$ & $f$ & 1.084 & 0.180 & 0.043 & 0.013 & 0.060 \\
\hline & TD-LC-DFTB// & $E$ & 3.195 & 3.796 & 3.957 & 4.290 & 4.294 \\
\hline & $\omega \mathrm{B} 97 \mathrm{XD}$ & $f$ & 1.187 & 0.219 & 0.000 & 0.038 & 0.099 \\
\hline & TD-LC-DFTB// & $E$ & 3.351 & 4.001 & 4.297 & 4.520 & 4.549 \\
\hline & LC-DFTB & $f$ & 1.394 & 0.070 & 0.000 & 0.000 & 0.031 \\
\hline \multirow{7}{*}{$\mathrm{PTBTBTz}_{2}$} & Method & & $\mathbf{S}_{1}$ & $\mathbf{S}_{\mathrm{B} 1}$ & $\mathbf{S}_{\mathrm{B} 2}$ & $\mathbf{S}_{\mathrm{B} 3}$ & $\mathbf{S}_{\mathrm{B} 4}$ \\
\hline & TD- $\omega \mathrm{B} 97 \mathrm{XD} / /$ & $E$ & 2.852 & 3.133 & 3.544 & 3.656 & 4.725 \\
\hline & $\omega B 97 X D$ & $f$ & 0.067 & 1.247 & 0.441 & 0.170 & 0.266 \\
\hline & TD-LC-DFTB// & $E$ & 3.049 & 3.262 & 3.472 & 3.564 & 3.799 \\
\hline & $\omega \mathrm{B} 97 \mathrm{XD}$ & $f$ & 0.095 & 1.380 & 0.177 & 0.111 & 0.343 \\
\hline & TD-LC-DFTB// & $E$ & 3.204 & 3.444 & 4.049 & 4.559 & 4.713 \\
\hline & LC-DFTB & $f$ & 0.043 & 2.247 & 0.169 & 0.121 & 0.165 \\
\hline
\end{tabular}

tion S-II), and approximate absorption cross sections are also shown. The face-on models have similar features regardless of the positions $(p=\mathrm{bt}, \mathrm{t}, \mathrm{btz})$ of the PCBM molecule. As shown in Table II, there are two low-lying absorption bands at $\approx 2.5 \mathrm{eV}$ and $\approx 2.8 \mathrm{eV}$ with oscillator strengths around $f \approx 2$ and $f \approx 1$, respectively (in case nearly degenerate states contribute to a given band, we refer to the sum of their strengths). For $p=\mathrm{bt}$, t, the bright states have LD character, although MX character for $p=$ btz. For the three systems, a significant density of LA states is found at the bottom of the bands, i.e., lying below the first bright state $(E \lesssim 2.55 \mathrm{eV})$. Only a few CT excitons are found either below the first bright state, close to the second bright state $(E \approx 2.8 \mathrm{eV})$, and between these two bright states $(2.55 \mathrm{eV} \lesssim E \lesssim 2.75 \mathrm{eV})$.

The models with $n=3$ stacked PTBTBTz dimers and one PCBM with face-on orien- 
tation, shown in Fig. 4(b), have denser spectra of LD states, as expected. Once more, the excited states were calculated for stationary structures, with no important differences arising from the different positions $(p=\mathrm{bt}, \mathrm{btz}, \mathrm{t})$ of the acceptor with respect to the donor (see also Table II). The cross sections show an absorption band around $2.9 \mathrm{eV}$, not present in the models with $n=2$ stacking (Fig. 4(a)), in addition to the bands around $2.5 \mathrm{eV}$ and $2.7 \mathrm{eV}$. The second band has the strongest optical coupling for the $n=3$ models, with bright states having either LD or MX character. Despite the differences in the absorption cross sections, the $n=2,3$ stacking models mostly have high-energy CT states, with only a few of such states lying around and below the brightest band.

The spectra of the $n=2,3$ stacking models with edge-on orientation are shown in Fig. 5 for the PCBM positions $p=\mathrm{bt}$, t. As described in the Supporting Informatiom (Section S-I), we performed MD simulations before obtaining the stationary structures. The edge-on models with $p=$ btz positions sometimes dissociated along the dynamics, so we did not consider interfaces combining $p=\mathrm{btz}$ and $o=e$. The absorption cross sections for the models with different orientations $(o=f, e)$ but same stacking are fairly similar, since the bright states mostly have LD character (see Figs. 4, 5, and Table II). However, the density of low-energy CT excitons is higher for the edge-on orientation, and this effect is more significant for $n=3$ stacking and $p=\mathrm{t}$ position. We further investigated the relation between interface orientation and CT excitons by selecting geometries from room-temperature MD simulations in the NVT ensemble, starting from the stationary structures. In all cases, including $n=2,3$ stacking, $p=\mathrm{bt}$, btz, t positions, and $o=f, e$ orientations, we considered 12 -fs trajectories and took either 10 ( $n=2$ stacking) or 5 ( $n=3$ stacking) randomly chosen geometries from the last 10 picoseconds. While the time span of the trajectories and the number of structures are admittedly insufficient to sample the thermodynamical ensemble, we consider initial conditions with different PCBM positions for each system, and long simulation times in the scale of the fast vibrational modes (stretching, bending). The procedure is hopefully adequate to indicate how robust the change in the density of low-energy CT excitons would be against temperature or fast geometry fluctuations, although keeping the computation time spent with MD simulations, excited state calculations and exciton analysis at a reasonable level.

As shown in the Supporting Information (Fig. S6), the absorption cross sections change significantly along the MD trajectories, and the oscillator strengths around $2.5 \mathrm{eV}, 2.7 \mathrm{eV}$, 


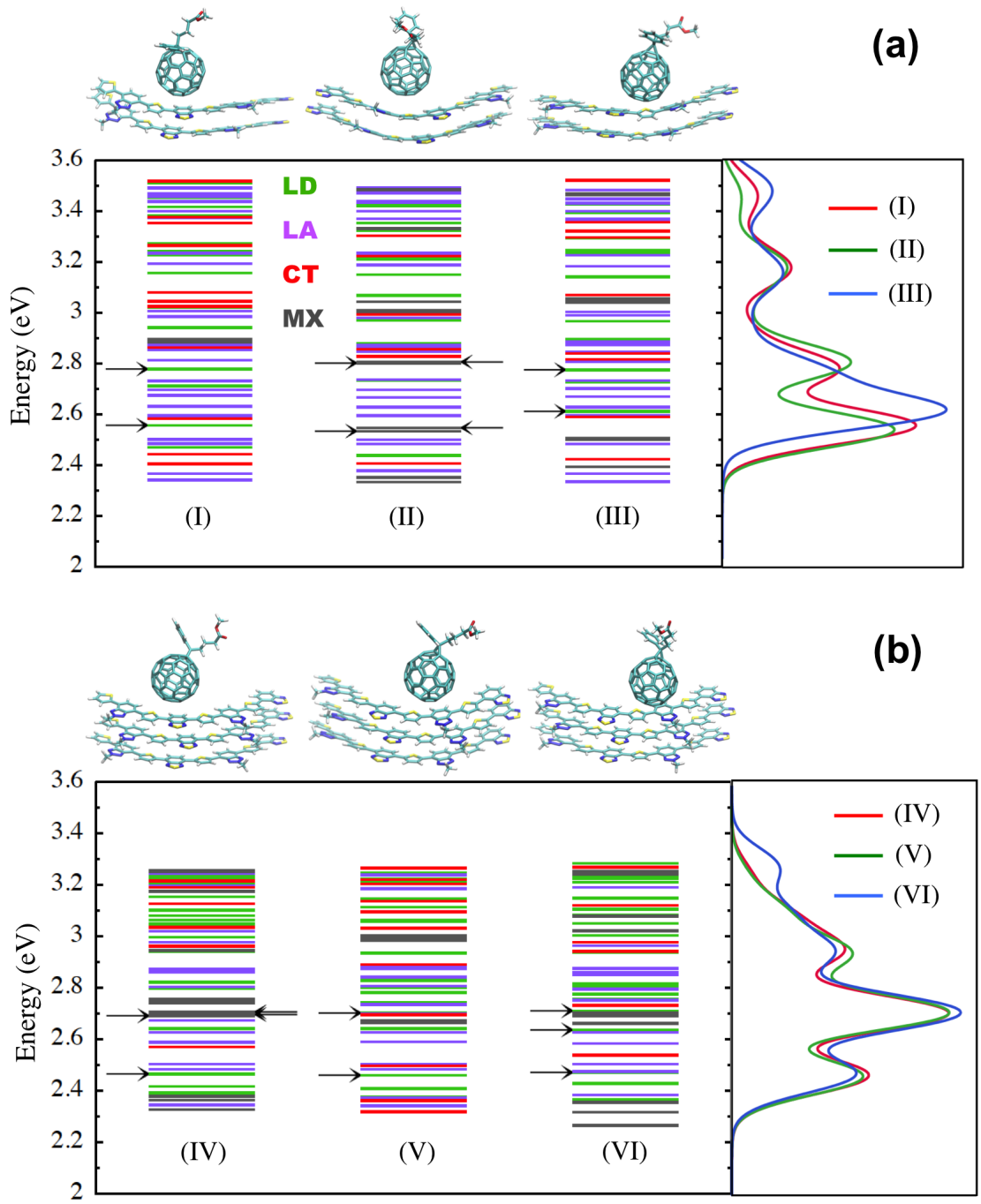

FIG. 4. (a) The main panel shows the vertical excitation spectra for the (dim-PT) $)_{2}:(\mathrm{PCBM} @ b t)_{1}: f$ (I), (dim-PT) $)_{2}:(\mathrm{PCBM} @ b t z)_{1}: f$ (II) and (dim-PT) $)_{2}:(\mathrm{PCBM} @ \mathrm{t})_{1}: f$ (III) systems. The LD states are indicated in green, LA in purple, CT in red, and MX in gray. The horizontal arrows point to the brightest transitions, and the geometries of the model systems are shown on top of the spectra. The right panel shows the absorption cross section (in arbitrary units) obtained from the broadening of the vertical spectra with Gaussian line shapes. (b) Same as in panel (a) for the $(\text { dim-PT) })_{3}:(\mathrm{PCBM} @ b t)_{1}: f(\mathrm{I}),(\text { dim-PT })_{3}:(\mathrm{PCBM} @ b t z)_{1}: f(\mathrm{II})$ and $(\text { dim-PT })_{3}:(\mathrm{PCBM} @ \mathrm{t})_{1}: f$ (III) systems.

and $2.9 \mathrm{eV}$ change by one order of magnitude among the different snapshots. Ideally, one should perform Monte Carlo integrations to obtain reasonably converged cross sections, but this procedure would request longer MD trajectories and probably $10^{3}$ to $10^{4}$ geometries, which would be unfeasible.

We defined cold CT excitons based on two criteria. For each system, we considered the 

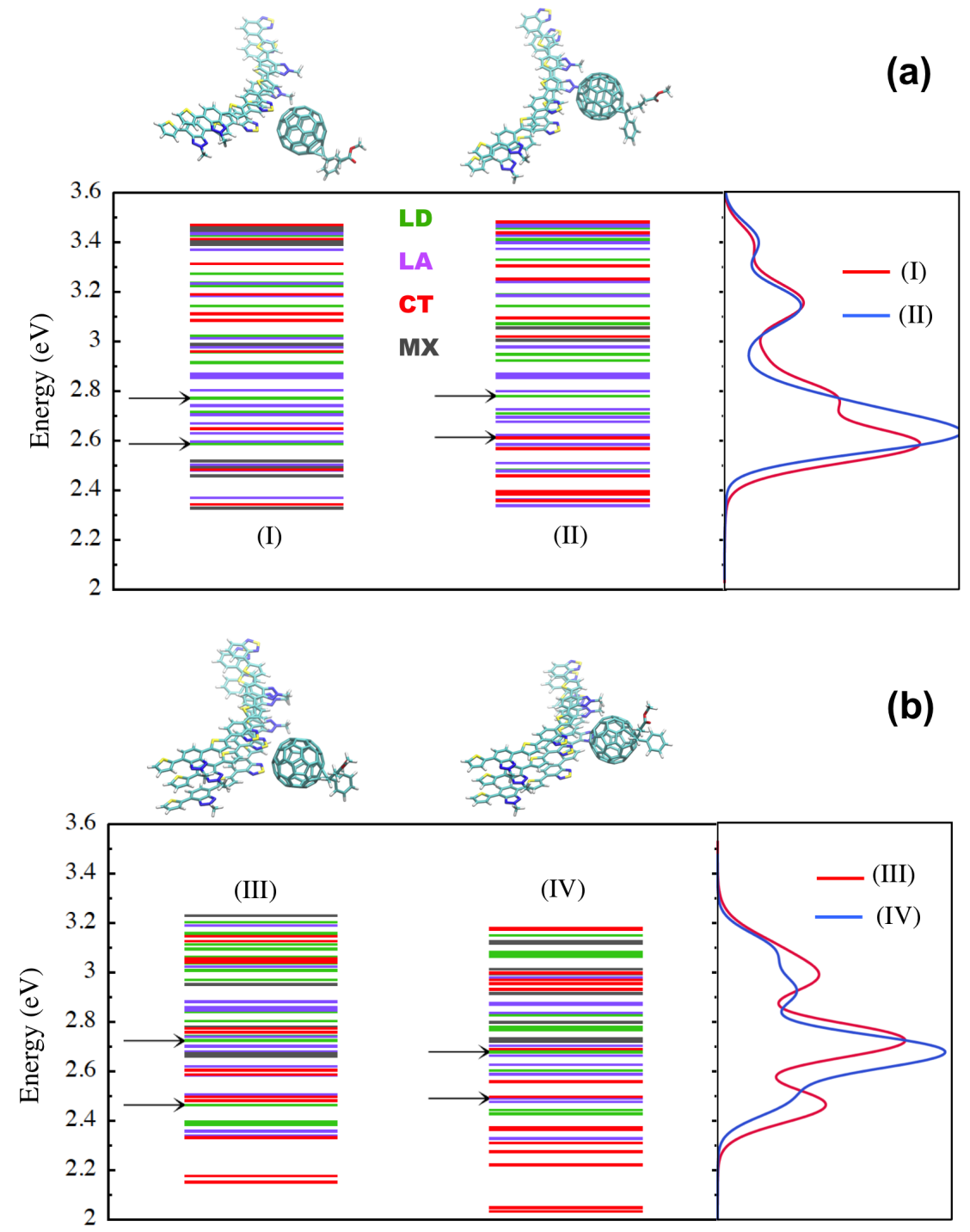

FIG. 5. (a) The main panel shows the vertical excitation spectra for the (dim-PT $)_{2}:(\mathrm{PCBM} @ b t)_{1}: e$ (I) and (dim-PT $)_{2}:(\mathrm{PCBM} @ \mathrm{t})_{1}: e(\mathrm{II})$ systems. The LD states are indicated in green, LA in purple, $\mathrm{CT}$ in red, and MX in gray. The horizontal arrows point to the brightest transitions, and the geometries of the model systems are shown on top of the spectra. The right panel shows the absorption cross section (in arbitrary units) obtained from the broadening of the vertical spectra with Gaussian line shapes. (b) Same as in panel (a) for the (dim-PT) $)_{3}:(\mathrm{PCBM} @ b)_{1}: e$ (III), and $(\text { dim-PT) })_{3}:(\mathrm{PCBM} @)_{1}: e(\mathrm{IV})$ systems.

CT states within a $0.2-\mathrm{eV}$ energy window (i) above the lowest-lying excited state with CT character, and (ii) above the $S_{1}$ state. The width of the energy window is of course arbitrary, but sizeable probabilities for non-adiabatic transition are expected for energy gaps around and below $0.2 \mathrm{eV}$, which justifies the present choice. The average number of cold CT states obtained from the criterion (i) and (ii), respectively denoted as $\left\langle n_{\mathrm{CT}}^{\text {cold }}\right\rangle$ and $\left\langle n_{\mathrm{CT}}^{\text {cold }}\right\rangle$, are shown 


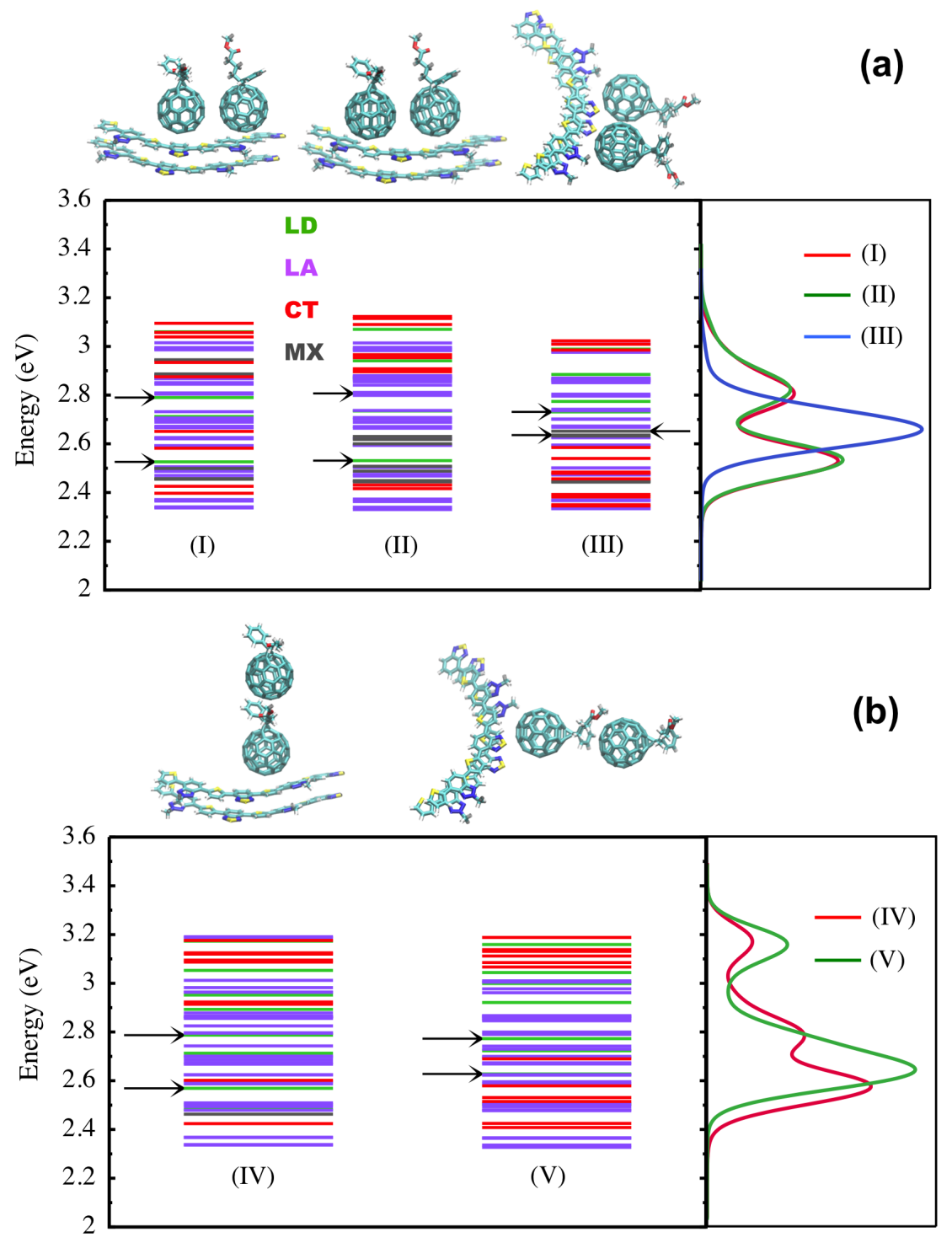

FIG. 6. (a) The main panel shows the vertical excitation spectra for the $(\text { dim-PT })_{2}:(\text { PCBM@bt_btz })_{2 s d}: f \quad(\mathrm{I}), \quad(\text { dim-PT) })_{2}:\left(\mathrm{PCBM} @ \mathrm{t} \_\mathrm{t}\right)_{2 s d}: f \quad(\mathrm{II}) \quad$ and $\quad$ (dim$\mathrm{PT})_{2}:\left(\mathrm{PCBM} @ \mathrm{t} \_\mathrm{t}\right)_{2 s d}: e$ (III) systems. The LD states are indicated in green, LA in purple, $\mathrm{CT}$ in red, and MX in gray. The horizontal arrows point to the brightest transitions, and the geometries of the model systems are shown on top of the spectra. The right panel shows the absorption cross section (in arbitrary units) obtained from the broadening of the vertical spectra with Gaussian line shapes. (b) Same as in panel (a) for the (dim-PT) $)_{2}:(\mathrm{PCBM} \mathrm{dt})_{2 r w}: f$ (IV), and $(\operatorname{dim}-\mathrm{PT})_{2}:(\mathrm{PCBM} @)_{2 r w}: e(\mathrm{~V})$ systems.

in Table III. The average number of CT states lying below the brightest ones, $\left\langle n_{\mathrm{CT}}^{\mathrm{low}}\right\rangle$, are also presented. In general, the different positions have a mild impact on the number of cold CT excitons, although $\left\langle n_{\mathrm{CT}}^{\text {cold }}\right\rangle,\left\langle n_{\mathrm{CT}}^{\text {cold } \star}\right\rangle$ and $\left\langle n_{\mathrm{CT}}^{\text {low }}\right\rangle$ tend to be smaller for $p=$ bt and larger for $p=\mathrm{t}$. The significance of these differences should be viewed with caution in view of 
the small number of geometries considered in the averages. The impact of orientation on the number of cold CT states is much clearer than that of position, confirming the trends suggested by the spectra of the stationary structures.

The excitation spectra of the models comprising two PCBM molecules are presented in Fig. 6. For the face-on orientation, we considered side-by-side arrangements in which the acceptors lie either on top of BT and BTz subunits ( $p=$ bt_btz) or on top of two T subunits $\left(p=\mathrm{t}_{\mathrm{t}} \mathrm{t}\right)$, while for the edge-on orientation we only considered the $p=\mathrm{t}_{\mathrm{t}} \mathrm{t}$ case to prevent dissociation during the MD. In panel (b) we show the results for the PCBMs arranged in a row at the $p=\mathrm{t}$ position. In view of the weak dependence of the calculated spectra on positions, the $p=$ bt case is shown in the Supporting Information (Fig. S5), while $p=$ btz models were not explored. It is clear from Fig. 6 and Tabs. II and III that the 2-PCBM models share the basic features with the single-PCBM counterparts. The spectra of CT states has a significant dependence on the orientation, although not as much on the position of the acceptor molecules. The bright states mostly have LD or MX character, despite the denser spectra of LA states. The edge-on orientation increases the density of cold CT excitons, an effect that is more evident for the $p=\mathrm{t}$ position than $p=\mathrm{bt}$, in case the PCBMs arranged in a row (see Fig. 6(b) and the Supporting Information, Fig. S5). Compared to the other interface models with edge-on orientation, the 2-PCBM systems with $2 \mathrm{rw}$ arrangement and $e$ orientation have more significant differences between $\left\langle n_{\mathrm{CT}}^{\text {cold }}\right\rangle$ and $\left\langle n_{\mathrm{CT}}^{\text {cold }}\right\rangle$. The reasons of this discrepancy, which indicates cold CT excitons lying further above the $S_{1}$ states, are not clear.

\section{DISCUSSION}

Our analysis has shown significantly larger densities of cold CT states in edge-on than in face-on interfaces. Such a density dependence on orientation has also been previously pointed out ${ }^{6,52}$ for other interface models with fullerene and non-fullerene acceptors. In the present work, however, we deliver a much in-depth picture of the CT exciton's dependence on the interface's architecture.

The orientation effect on the cold CT states seems to be a general property of DA interfaces $^{6,47,51,52,54}$ and can be further investigated by the fragment-based analysis outlined in Sec. II. We averaged the CT numbers, defined in eq. 4, for the different models using the 
geometries sampled from the MD simulations. We considered the cold CT states lying up to $0.2 \mathrm{eV}$ above the $S_{1}$ state, such that the number of data points in each average is given by $N \times\left\langle n_{\mathrm{CT}}^{\text {cold }}\right\rangle$, where $N$ is the number of structures and $\left\langle n_{\mathrm{CT}}^{\text {cold }}\right\rangle$ is the average number of cold CT states. In addition to increasing the spectral density of low-energy CT states, the edge-on orientation enhances the level of charge separation. In nearly all cases, one finds $\left\langle\mathrm{CT}_{\text {cold }}\right\rangle<0.9$ and $\left\langle\mathrm{CT}_{\text {cold }}\right\rangle \geq 0.95$ for the face-on and edge-on models, respectively, regardless of the oligomer stacking as well as the number, position, and arrangement of the PCBM molecules. Although not shown here, a similar trend was found for the cold CT states defined with respect to the lowest-lying CT exciton, and also for the CT states lying below the brightest states. The CT states at the bottom of the excited state spectra are believed to dissociate thermally ${ }^{5,6}$, a process that should be favored by edge-on orientation given the increased interfacial charge transfer and the higher density of cold CT states ${ }^{6}$.

Further insight into the orientation effect on the CT excitons can be gained from the analysis of the POS descriptor, defined in eqs. 5 and 6 . The fragmentation scheme follows the conventions shown in Figs. 7(b) and 8(b) for the models with $n=2,3$ stacking, respectively. For a decomposition into $N_{\text {fr }}$ fragments, the oligomer chains are labeled $A=1$ to $A=$ $\left(N_{\text {fr }}-1\right)$, from the furthest- to the closest-lying one with respect to the PCBM molecule, which is labeled $A=N_{\text {fr }}$. While the positions might not be as meaningful for the edge-on orientation, since the fragments are not aligned, the POS descriptor can still be interpreted as a weighted contribution from the fragments to the excitons. The POS descriptor for the CT states of the $n=2$ stacking models are presented in Fig. 7(a) as a function of the excitation energy. We only show the data points for the stationary structures to avoid overloading the panel, but the results are representative. The vertical shaded areas indicate the energy range of the bright states, where the brightest ones lie around $\approx 2.55 \mathrm{eV}$. The $\mathrm{CT}$ numbers, given in the color map, were obtained for two fragments, corresponding to the donor and acceptor domains, for consistency with the previous results (this procedure also avoids that a LD state involving charge transfer between the oligomer chains be assigned as a CT exciton). From eqs. 5 and 6 , a CT exciton with the hole fully localized on the fragment $A=1$ and the electron fully localized on the fragment $A=3$ will have POS $=2.00$. Accordingly, POS $=2.50$ results from hole localization on the $A=2$ fragment, while POS $=2.25$ from an evenly delocalized hole over the $A=1,2$ fragments. These ideal cases are also indicated by the dashed lines. The cold excitons of the structures with face-on orientation essentially 
(a)

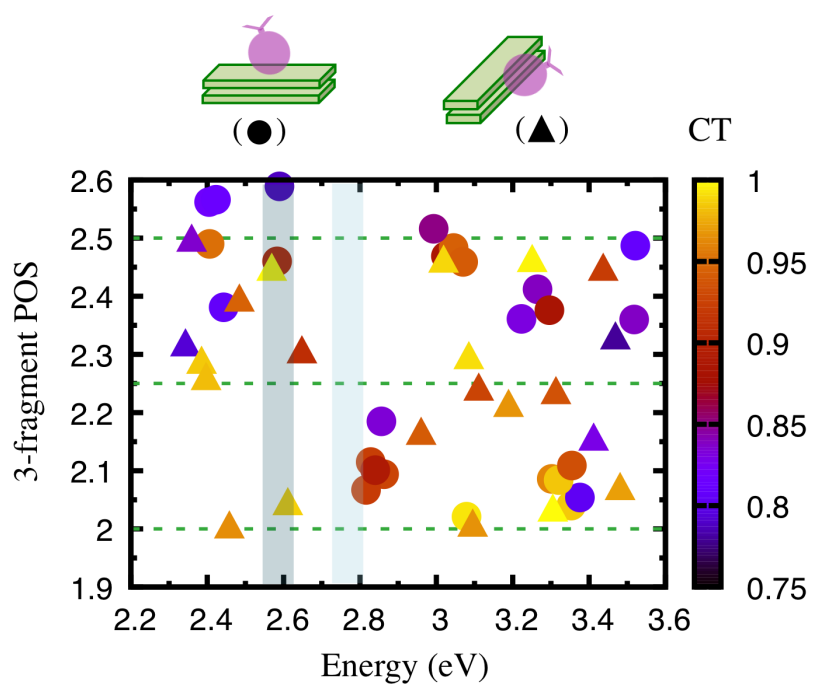

(b)
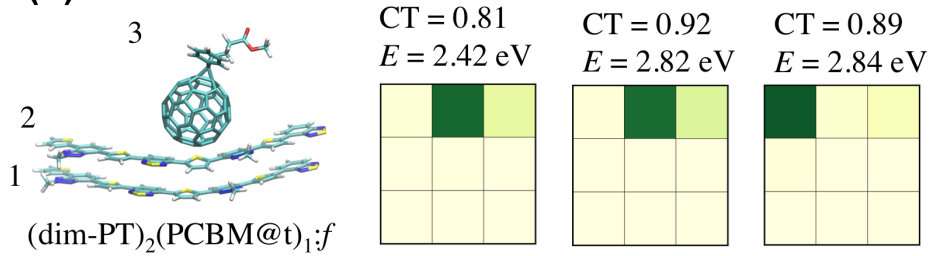

Iow

high

high
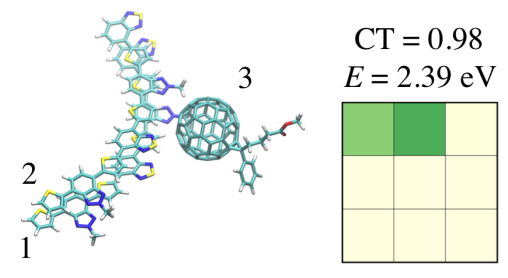

$(\operatorname{dim}-\mathrm{PT})_{2}(\mathrm{PCBM} @ \mathrm{t})_{1}: e$

low

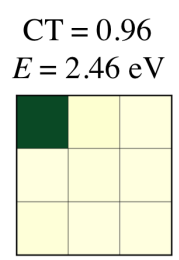

low

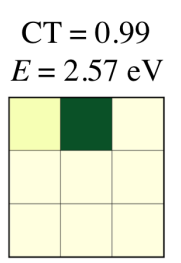

low

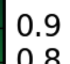

0.8
0.7

0.6

$-0.5$

$-0.4$

$-0.3$

$-0.2$

$-0.1$

FIG. 7. (a) Position (POS) descriptor for the CT states as a function of the excitation energy. The color map indicates the corresponding CT numbers. The data points were obtained for the stationary structures of the interface models with $n=2$ stacked chains in the donor domain, (dim$\mathrm{PT})_{2}:(\mathrm{PCBM} @ p)_{1}: f$ (circles) with $p=$ bt, btz,t, and (dim-PT) $)_{2}:(\mathrm{PCBM} @ p)_{1}: e$ (triangles) with $p=\mathrm{bt}$, t. The POS numbers were computed for the three-fragment decomposition shown on the left-hand side of the panel (b), although the CT numbers were obtained from a two-fragment (DA) decomposition scheme. The horizontal dashed lines correspond to the POS values of ideal CT states with the hole localized on fragment 1 ( $\mathrm{POS}=2.00)$, localized on fragment $2(\mathrm{POS}=2.50)$, or evenly delocalized over fragments 1 and $2(\mathrm{POS}=2.25)$. The shaded regions indicate the energy range where the bright states are found (the brightest states of the five structures lie at $\approx 2.55 \mathrm{eV}$ ). (b) The structures of the (dim-PT) $)_{2}:(\mathrm{PCBM} @ \mathrm{t})_{1}: o$ models, with $o=f, e$ orientations, are shown on the left-hand side along with the fragmentation convention. Electron-hole correlation plots for selected CT states are presented on the right-hand side, following the convention given in Fig. 1(c). The CT states are indicated as "high" and "low" in case they lie above or below the brightest state.

arise from $(A=2 \rightarrow B=3)$ transitions, where $A$ and $B$ denote, respectively, the fragments where the hole and the electron reside. The POS descriptor of those CT excitons also 
exceeds the limiting value of 2.50, which indicates the admixture of LA character, consistent with the relatively low CT numbers, below 0.85. Only the high-energy CT excitons, lying above $2.8 \mathrm{eV}$, display significant charge transfer from the $A=1$ fragment, although the POS descriptors for excitons with similar excitation energies spread considerably between the limits the ideal $(1 \rightarrow 3)$ and $(2 \rightarrow 3)$ transitions. These trends are consistent with TDDFT studies on interfacial CT states of DTDCTB: $\mathrm{C}_{60}$ models ${ }^{46}$ with face-on orientation. In the cold CT states, the hole mainly localizes on a single DTDCTB chain, also lying further from the hole as the energy of the CT state increases. Co-facial pentacene- $\mathrm{C}_{60}$ systems with face-on orientation ${ }^{47}$ also display more significant delocalization of the hole among the stacked donor chains, and also larger electron-hole distances in larger models containing four pentacene molecules.

For the structures with edge-on orientation, one finds $2.00<\mathrm{POS}<2.50$ for both cold and hot excitons, pointing out that charge transfer from either (or both) donor fragments can take place with no clear dependence on the photon energy. The electron-hole correlation plots for a few representative CT excitons of the (dim-PT) ${ }_{2}(\mathrm{PCBM} @)_{1}: f$ and $(\text { dim-PT })_{2}(\mathrm{PCBM} @)_{1}: e$ models are shown in Fig. 7(b). The convention for the hole and electron axes are defined in Fig. 1(c). The low-energy CT exciton of the face-on structure $(2.42 \mathrm{eV})$ mostly arises from the $(2 \rightarrow 3)$ transition, while in the high-energy states, the hole resides either on the $A=1(2.82 \mathrm{eV})$ or $A=2(2.84 \mathrm{eV})$ donor fragments. The admixture of LA character is fairly clear for the two lowest-lying states (green shade on the $A=B=3$ matrix element). The edge-on structures show low-energy CT excitons formed by transfer out of either donor fragment $(2.46 \mathrm{eV}$ and $2.57 \mathrm{eV})$ or both of them $(2.36 \mathrm{eV})$. The lack of noticeable LD or LA admixture is also consistent with the more effective charge transfer, indicated by $\mathrm{CT}>0.95$.

The POS descriptors for the stationary structures of the models with $n=3$ stacking are shown in Fig. 8(a). While the general trends are shared with the $n=2$ models, some aspects are noteworthy. Despite the more thorough charge transfer, with $\mathrm{CT} \approx 0.90$ and a smaller admixture of LD charater, the models with face-on orientation still show cold CT excitons with holes mostly localized on the donor fragment lying the closest to the PCBM molecule. It should be clear that, according to the decomposition into 4 fragments, the ideal $(1 \rightarrow 4)$ and $(3 \rightarrow 4)$ transitions result in $\mathrm{POS}=2.50$ and $\mathrm{POS}=3.50$, respectively, while POS $=3.00$ reflect either the ideal $(2 \rightarrow 4)$ transition, hole delocalization over non- 
(a)

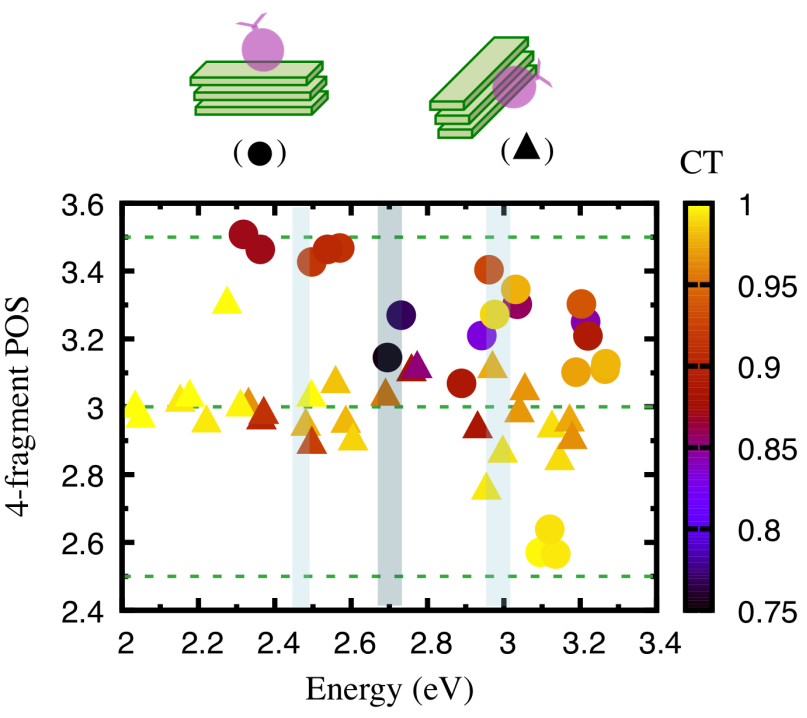

(b)
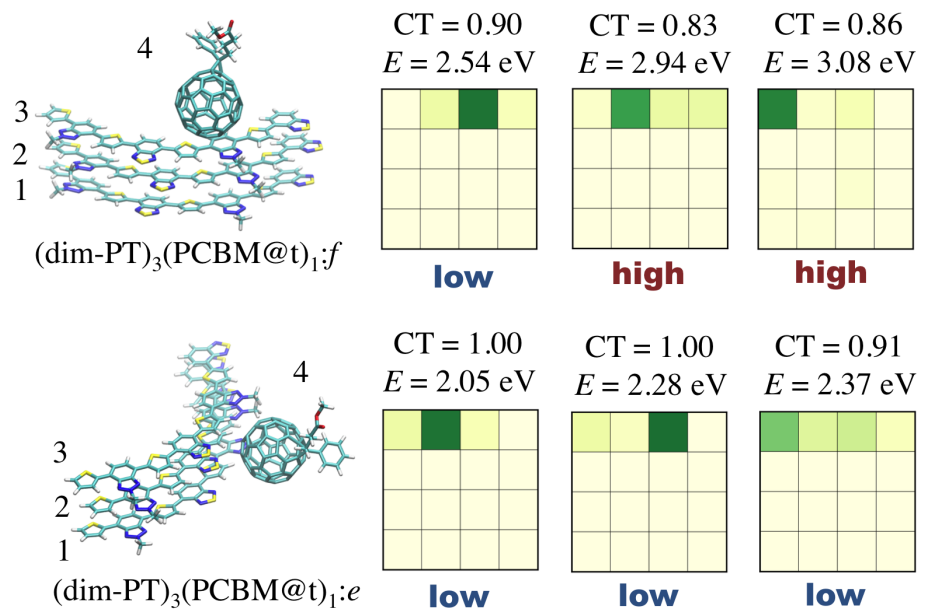

Iow

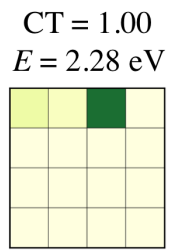

Iow

0.9

0.8

0.7

0.6

0.5

$\mathrm{CT}=0.91 \quad-0.4$

$E=2.37 \mathrm{eV} \quad-0.3$

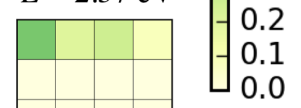

FIG. 8. (a) Position (POS) descriptor for the CT states as a function of the excitation energy. The color map indicates the corresponding CT numbers. The data points were obtained for the stationary structures of the interface models with $n=3$ stacked chains in the donor domain, (dim$\mathrm{PT})_{3}:(\mathrm{PCBM} @ p)_{1}: f$ (circles) with $p=\mathrm{bt}$, btz,t, and (dim-PT) $)_{3}:(\mathrm{PCBM} @ p)_{1}: e$ (triangles) with $p=\mathrm{bt}$, t. The POS numbers were computed for the four-fragment decomposition shown on the left-hand side of panel (b), although the CT numbers were obtained from a two-fragment (DA) decomposition scheme. The horizontal dashed lines correspond to the POS values of ideal CT states with the hole localized on fragment 1 ( $\mathrm{POS}=2.5)$, localized on fragment 3 ( $\mathrm{POS}=3.5)$, and either localized on fragment 2 or evenly delocalized over fragments 1,2 and 3 (POS = 3.0). The shaded regions indicate the energy range where the bright states are found (the brightest states of the five structures lie at $\approx 2.7 \mathrm{eV})$. (b) The structures of the (dim-PT) $3:(\mathrm{PCBM} @)_{1}: o$ models, with $o=f, s$ orientations, are shown on the left-hand side along with the fragmentation convention. Electron-hole correlation plots for selected CT states are presented on the right-hand side, following the convention given in Fig. 1(c). The CT states are indicated as "high" and "low" in case they lie above or below the brightest state.

neighboring fragments, $(1,3 \rightarrow 4)$, or uniform delocalization of the hole, $(1,2,3 \rightarrow 4)$. Only 
hot CT states lying above the third absorption band have prevailing $(1 \rightarrow 4)$ character. These high-energy excitons with large electron-hole separations also show the most effective charge transfer, with $\mathrm{CT} \approx 1$, although in general, one finds $\mathrm{POS}>3.0$ and $\mathrm{CT} \approx 0.9$ for the face-on models. The electron-hole correlation plots for representative CT excitons of the (dim-PT) $)_{3}:(\mathrm{PCBM} @ \mathrm{t})_{1}: f$ structure are shown in Fig. 8(b). In contrast, the edge-on orientation mostly gives rise to $\mathrm{POS} \approx 3$, suggesting more effective hole delocalization over the donor chains, according to the ideal transitions described above. Only for high-energy CT excitons, around and above the third band, the values of the position descriptors are more spread. In general, the charge transfer is more effective, as previously pointed out, and the hole delocalization is also clear from the correlation plots of the representative CT excitons, shown in Fig. 8(b).

The POS descriptor is expected to correlate with the exciton size $\left(d_{\text {exc }}\right.$, see eqs. 7 and 8 ), which would significantly impact the dissociation of cold interfacial excitons ${ }^{50}$. The connection between exciton size and the average positions of the hole and electron (POS, see eq. 6) might be affected by several aspects, such as the degree of (de)localization of the particles within a given fragment, the fragmentation scheme employed to define the POS descriptors, and the detailed structure of the 1-TDM. The approximations underlying eq. 8, particularly the definition of atomic volumes with constant electron-hole distances for the atomic pairs, might also impact the results. While we did not explore more complex fragmentation schemes to investigate the quasi-particle delocalization in detail, $d_{\text {exc }}$ is shown in Fig. 9(a) for the CT states of the models with $n=3$ stacking (stationary structures). The exciton size is plotted against the POS descriptor obtained from four fragments as before, and also the two-fragment CT number, indicated by the color map. Different positions are not discriminated, so we focus on the orientations. The face-on models (circles) display a stronger $d_{\text {exc }}$ dependence on position since the (de)localization of the hole over different donor chains has a clear effect on the charge separation, with the electron essentially localized on the PCBM molecule. In case the hole is localized on the $A=3$ fragment, which is the oligomer chain lying the closest to the acceptor $(\mathrm{POS} \approx 3.5)$, we obtain $d_{\text {exc }} \approx 9.5 \AA$. For the CT states with POS $\approx 2.5$, consistent with $(1 \rightarrow 4)$ transitions, one finds $d_{\text {exc }} \approx 14.5 \AA$. As discussed above, the localization of the hole on the $A=3$ and $A=1$ fragments typically produces low- and high-energy CT excitons, respectively. A few ideal excitations might be of help to better understand the intermediate values of the POS descriptor. The even 
delocalization of the hole over two fragments leads to $(1,2 \rightarrow 4)$ and $(2,3 \rightarrow 4)$ transitions, and hence to $\mathrm{POS}=2.75$ and $\mathrm{POS}=3.25$, respectively. As mentioned above, $\mathrm{POS}=3.00$ can arise from either $(2 \rightarrow 4),(1,3 \rightarrow 4)$ or $(1,2,3 \rightarrow 4)$ ideal excitations. Most of the excited states have POS $>3.00$, with the degree of charge transfer generally increasing with the exciton size. As a rule of thumb, face-on models give rise to CT excitons that increase in size and charge separation as a function of the excitation energy, according to the results shown in Figs. 8(a) and 9(a).

The edge-on models (triangles) do not show a clear relation between position and excitation energy, as discussed above. The $d_{\text {exc }}$ dependence on position is not evident, either, since nearly all points lie within $(2.75<$ POS $<3.25)$. This suggests hole delocalization over the donor chains, although with no clear trend, since several ideal transitions have POS descriptors lying in that range. Also as a rule of thumb, the exciton size increases with the degree of charge transfer and the excitation energy, as inferred from Figs. 8(a) and 9 (a). Inspection of the electron-hole correlation plots (not shown) indicates that the largest excitons arise from excitations having either dominant $(1,3 \rightarrow 4)$ or $(1,2,3 \rightarrow 4)$ characters. While it makes physical sense that these transitions produce large excitons, as they maximize the delocalization of the hole, the present analysis provides limited information since smaller CT excitons have similar fragment contributions. Breaking the interface models into more fragments could provide more detailed information, but we do not pursue this goal in the present work. In any case, exciton sizes as large as $d_{\mathrm{exc}} \approx 15 \AA$ are found for both orientations, although the electron-hole characteristics are not the same. Apart from the delocalization along the chains, the face-on models produce larger excitons by placing the hole on oligomer chains lying far from the acceptor, whereas larger excitons are produced by the delocalization of the hole in edge-on models.

The delocalization of the hole along the oligomer chains was further explored with models composed of PTBTBTz tetramers, namely (tet-PT) $)_{2}(\mathrm{PCBM} @ p)_{1}: f$, and also PT3BTBTz dimers, (dim-PT3) ${ }_{2}(\mathrm{PCBM} @ p)_{1}: f$. The data in Fig. 9(b), obtained for stationary structures, spread considerably, such that exciton sizes around and below $14 \AA$ are found for the face-on models built on (dim-PT) $)_{2}$, (tet-PT) $)_{2}$ and (dim-PT3) $)_{2}$ donors. Nevertheless, larger excitons are produced by the two larger models, as expected, and the trend of size increase with the CT number is once more noticeable. Despite the limited number of data points, the largest excitons $\left(15 \AA<d_{\text {ext }}<18 \AA\right)$ are mostly built on PT3BTBTz dimers. In general, 

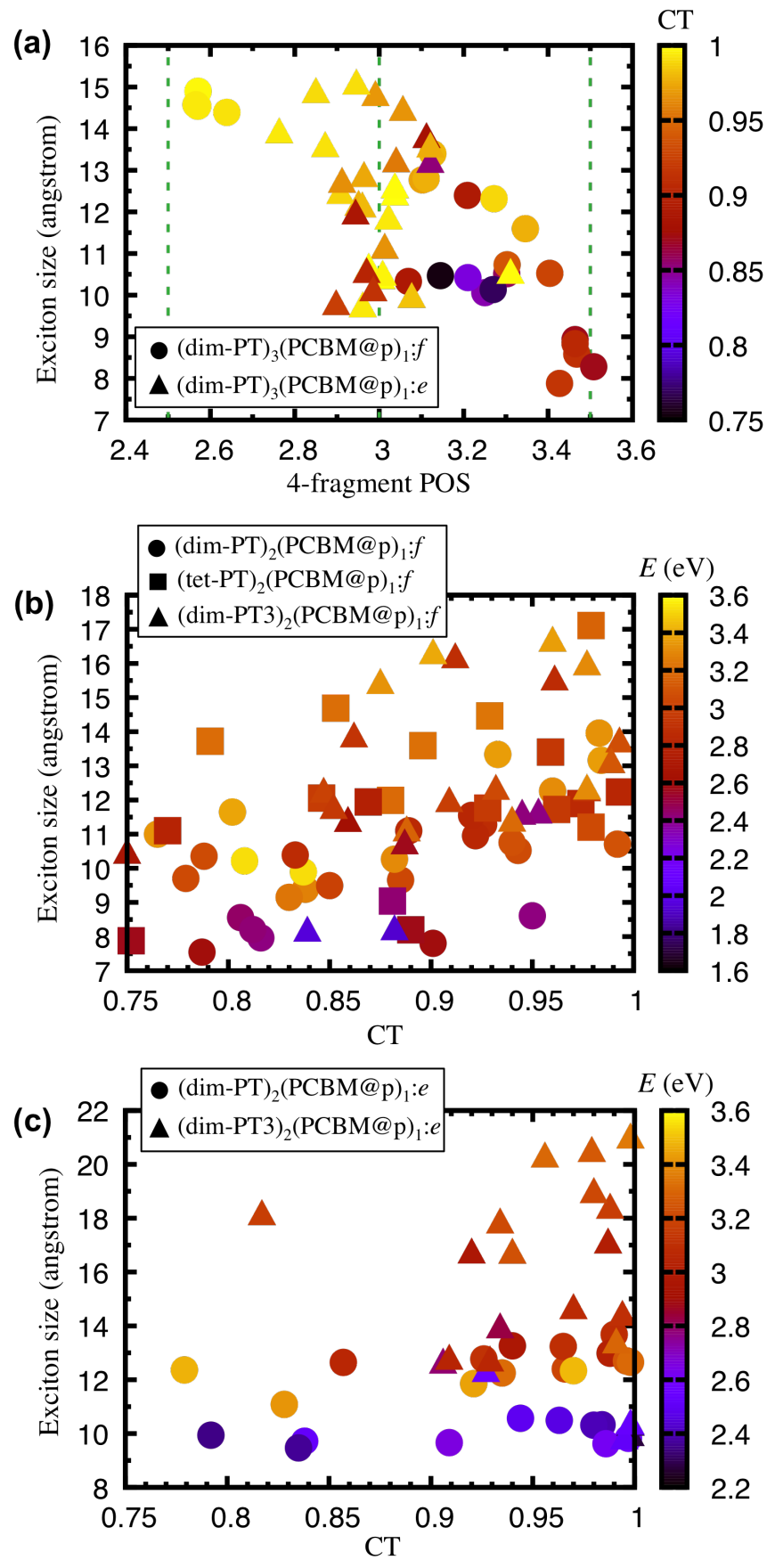

FIG. 9. (a) Exciton size against the position descriptor (POS) and the charge transfer number $(\mathrm{CT})$, given in the color map, for the interface models indicated in the panel. In each case we consider different positions of the PCM molecule: $\mathrm{p}=\mathrm{bt}$, btz, $\mathrm{t}$ (circles); $\mathrm{p}=\mathrm{bt}, \mathrm{t}$ (triangles). The vertical lines indicate the POS descriptor values for ideal excitations (see Fig. 8(a)). (b) Exciton size against the charge transfer number (CT) and the excitation energy, given in the color map, for the face-on interface models indicated in the panel. The PCBM positions are $\mathrm{p}=\mathrm{bt}$, btz (circles); $\mathrm{p}=\mathrm{bt}, \mathrm{t}$ (squares); $\mathrm{p}=\mathrm{bt}$, btz, $\mathrm{t}$ (triangles) (c) Same as in panel (b), for the edge-on models with positions $\mathrm{p}=\mathrm{bt}, \mathrm{t}$ (circles and triangles). 
the (dim-PT3) $)_{2}$ donors remain more planar during MD and geometry optimization than the (tet-PT) $)_{2}$ counterparts (see the structures in the Supporting Information, Figs. S2 and S3), which favors the delocalization of the molecular orbitals and hence the delocalization of the hole. The exciton sizes for the edge-on models built on (dim-PT) $)_{2}$ and (dim-PT3) 2 donors are presented in Fig. 9(b). The latter donors produce larger excitons with more thorough charge separation. We mention in passing that the PT3BTBTz:PCBM OSCs are more efficient than the PTBTBTz:PCBM ones. While we cannot draw conclusions on the efficiencies of the devices from the present models, the atomistic simulations point out two aspects that could contribute to the higher efficiency of the PT3BTBTz:PCBM cells, namely the stiffer donor chains that favor exciton delocalization and the larger oscillator strengths (between $\approx 4$ and $\approx 6$ for the brightest bands). Finally, the edge-on PT3BTBTz models produce considerably larger excitons, $18 \AA<d_{\text {exc }}<22 \AA$, than the face-on counterparts. This is in contrast with the $(\text { dim-PT) })_{3}$ models, with similar exciton sizes for both orientations. One must notice, however, that the $n=2$ stacking poses a limit for electron-hole separation in the face-on (dim-PT3) 2 donors, preventing the formation of larger excitons.

We now turn attention to the delocalization of the electron, exploring the interface models with two PCBM molecules. The exciton sizes of the CT states are shown in Fig. 10 for the systems with the acceptor units arranged side by side. For the face-on orientation (circles), the CT excitons lying around and below the second absorption band $(\lesssim 2.8 \mathrm{eV})$ have small sizes, $d_{\text {exc }}<9.0 \AA$, since they are essentially given by $(2 \rightarrow 3)$ and $(2 \rightarrow 4)$ transitions, according to the fragment labels indicated in the top panel. The values of the POS descriptor spread considerably because the charge can be transferred to either PCBM molecule $(A=3$ or $A=4)$. In our calculations, the electron did not delocalize over the two acceptor units, even for the higher-energy CT states, such that the largest exciton sizes, $d_{\text {exc }} \approx 12 \AA$, mostly correspond to $(1 \rightarrow 3)$ and $(1 \rightarrow 4)$ transitions, as indicated by the selected correlation plots in Fig. 10. The two PCBM molecules arranged side by side do not give rise to larger excitons compared to the analogous models, built on (dim-PT) 2 donors, with a single PCBM molecule (see Fig. 9(b)). This result is consistent with TDDFT computations for pentacene: $\mathrm{C}_{60}$ interface models ${ }^{36}$. Only the larger systems, comprising three $\mathrm{C}_{60}$ units, have low-energy $\mathrm{CT}$ states with electron delocalization over the acceptor molecules. The negative charge remains localized on a single $\mathrm{C}_{60}$ for smaller models with up to two molecules in the acceptor domain. 


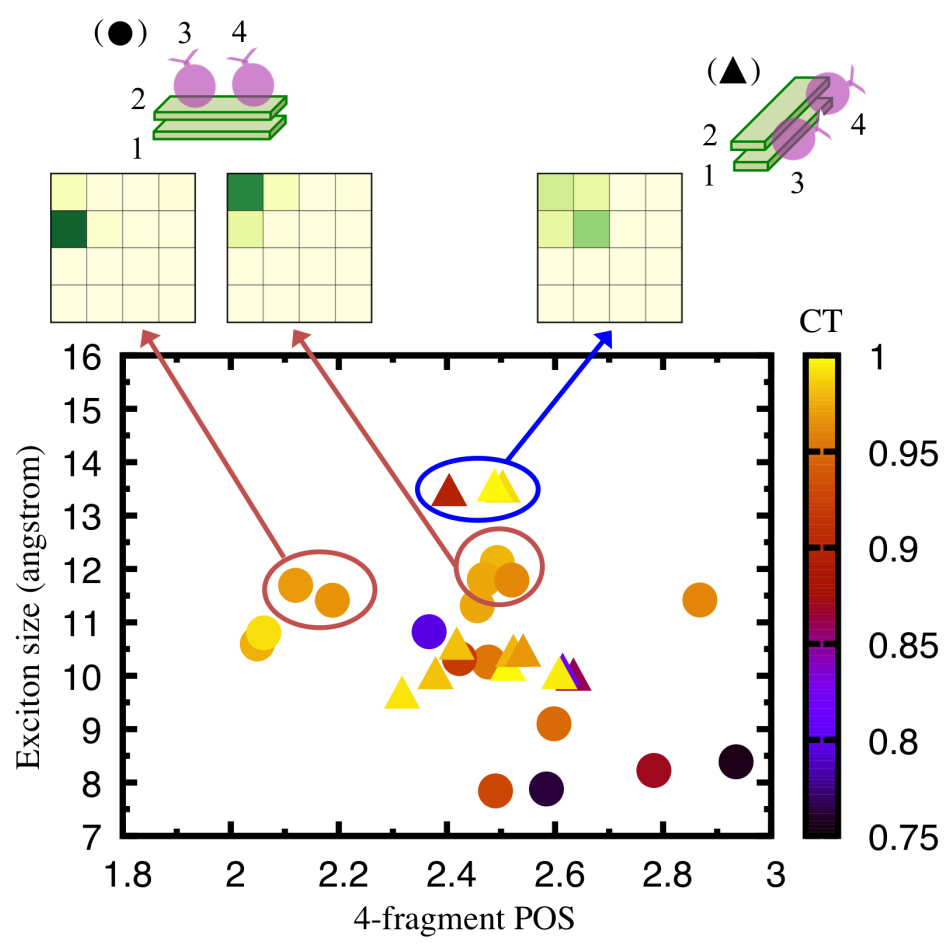

FIG. 10. Exciton size against the position descriptor (POS) and the charge transfer number $(\mathrm{CT})$, given in the color map, for the interface models (dim-PT) $)_{2}(\mathrm{PCBM} @ \mathrm{p})_{2 s d}: f$ (circles) and (dim-PT $)_{2}(\mathrm{PCBM} @ \mathrm{p})_{2 s d}: e$ (triangles). In both cases, the data points include the $\mathrm{p}=$ bt_btz,t_t positions. representative electron-hole correlation plots are shown above and below the main panel, with the fragment labels (1 to 4 ) indicated on the top.

In the case of edge-on orientation (triangles), we mostly obtained delocalized holes for the lower-lying CT excitons produced by $(1,2 \rightarrow 3)$ or $(1,2 \rightarrow 4)$ transitions, while both the hole and the electron tend to delocalize for the higher-energy CT states. The fully delocalized high-energy CT states are illustrated by the correlation plot in Fig. 10, having $d_{\text {exc }} \approx 13.5 \AA$. As for the face-on orientation, the addition of a PCBM molecule does not significantly alter the sizes of the largest excitons compared to the similar edge-on models with a single PCBM molecule (not shown). This happens because the delocalization of the electron is compensated by the smaller electron-hole distances.

For the models with two PCBM molecules arranged in a row, the dependence of the CT exciton sizes with respect to the POS descriptor and CT number is presented in Fig. 11, along with electron-hole correlation plots for representative states. Once more, we do not distinguish the positions and concentrate on the orientations. The CT states of the face-on systems (circles) fall into three distinct regions. The low-energy excitons are typically formed 




FIG. 11. Exciton size against the position descriptor (POS) and the charge transfer number (CT), given in the color map, for the interface models (dim-PT) ${ }_{2}(\mathrm{PCBM} @ \mathrm{p})_{2 r w}: f$ (circles) and (dim$\mathrm{PT})_{2}(\mathrm{PCBM} @ \mathrm{p})_{2 r w}: e$ (triangles). In both cases, the data points include the $\mathrm{p}=\mathrm{bt}$, t positions. Representative electron-hole correlation plots are shown above and below the main panel, with the fragment labels (1 to 4 ) indicated on the left.

by $(2 \rightarrow 3)$ transitions, where the donor fragments are labeled 1 and 2 , while the acceptor fragments, 3 and 4, as indicated in the panel. Ideally, those transitions are characterized by $\mathrm{POS}=2.5$. The CT excitons with POS $=2.0$ are mostly formed out of $(1 \rightarrow 3)$ transitions, having intermediate energies, while only at the higher energies we observe significant electron delocalization on the $A=4$ fragment, corresponding to the PCBM molecule lying further from the donor domain. In the latter case, the exciton sizes significantly increase to $d_{\text {exc }} \approx$ $21 \AA$. Although the data points are somewhat scattered, the edge-on models produce larger low-energy excitons than the face-on counterparts, as they allow for the delocalization of the hole over the oligomer chains. Only for the high-energy CT excitons, however, the electron delocalizes over the far-lying PCBM molecule. This combination of a delocalized hole (over the two stacked chains) and a distant electron produces the largest CT states among all the models explored in the present study, with $d_{\text {exc }} \approx 24 \AA$.

The delocalization of the interfacial CT excitons is, of course, limited by the system sizes, 
but some of the trends are compatible with those obtained from tight-binding models for much larger interfaces ${ }^{6}$. In the latter models, the higher-energy CT states have a band-like structure, approaching charge-separated states. On average, the electron-hole distances are smaller for the model in which the hole tends to delocalize along the DA interface. This result is in line with the present ones for the models having two PCBM molecules arranged side by side since the high-energy exciton sizes are not particularly large even when delocalized over all fragments. In case the acceptor molecules are arranged in a row, the delocalization of the high-energy CT excitons is more perpendicular to the interface, producing larger electronhole distances. From the photophysics perspective, the present study points out to similar trends as the previous TD-DFT study on P3HT:PCBM models ${ }^{52}$. Hot CT states, having energies around the absorption bands, would be expected to play a more significant part in interfaces with face-on orientation. Cold CT excitons, lying close to the $S_{1}$ state, would be more relevant to those with edge-on orientation. Population transfer to cold excitons is favored by the higher density of those states and also by the cascade of CT excitons lying below the brightest states (see Fig. 5 and 6). Once hot CT excitons are populated from the bright states, relaxation to lower CT states would require smaller electronic rearrangement, compared to LD and LA states, such that stronger diabatic couplings could be expected.

Finally, the role of entropic forces arising from the density of electronic states in the dissociation of hot $\mathrm{CT}$ excitons has been recently pointed out ${ }^{6}$, but we cannot explore those entropy effects based on the present models.

\section{CONCLUSIONS}

We have employed the TD-LC-DFTB method and fragment-based 1-TDM analysis to study the CT states of polymer:fullerene interfaces, exploring models of different sizes, as well as different positions and orientations of the acceptor molecules with respect to those of the donor domain. The orientation effect is significant since the edge-on models have denser spectra of cold CT excitons, lying below the absorption bands. This effect was observed for all models addressed in the present study, taking into consideration both the stationary structures and the geometries obtained from MD simulations. The interface orientation is thus expected to impact the excited state dynamics and charge separation mechanisms.

Phases with face-on orientation favor dissociation from hot excitons, lying around the 
brightest bands. Edge-on phases favor dissociation following relaxation to cold CT states, which is enhanced by the cascade of CT excitons. These photophysical mechanisms, summarized in Fig. 12, are consistent with previous atomistic simulations ${ }^{52}$. The exciton analysis also points out to the formation of low-energy interfacial CT states with increased electronhole separation in the edge-on phases. This effect would also make the charge separation from cold CT states more efficient, along with the higher density of these low-energy states.

The position of the acceptor molecules with respect to the donor is generally less important, although the change in the excitation spectra from the face-on to the edge-on orientation tends to be more clear when the PCBM molecule lies closer to the T subunit of the donor chains. The face-on orientation gives rise to fewer cold CT states, which also have, on average, smaller CT numbers compared to the cold CT states in the models with edge-on orientation. The degree of charge transfer and the CT exciton size tends to increase with the excitation energy. In the edge-on models with a single PCBM molecule, the charge transfer is favored by longer electron-hole distances arising from hole localization on donor chains lying far from the acceptor molecules, while hole delocalization over different donor chains prevails in the edge-on interfaces. The models with two acceptor molecules suggest that the electron remains localized on a single molecule in the cold CT states. The delocalization over two PCBM molecules was observed for higher-energy CT states, although the impact on the exciton size also depends on the orientation. Larger excitons are produced by the delocalization of the electron perpendicularly to the DA interface. In case the delocalization is parallel to the interface, it is compensated by the smaller electron-hole distances, producing moderately sized CT excitons.

\section{SUPPORTING INFORMATION}

Details of the MD simulations and geometry optimization. Structures of the PTBTBTz stacked dimer, the PT3BTBtz monomer, and also of the interface models not shown in the main text. Photo-absorption cross sections for the PTBTBTz monomer calculated with the nuclear ensemble method. Vertical excitation spectra of the (dim-PT) $)_{2}:(\mathrm{PCBM} @ b t)_{2 \mathrm{rw}}: f$ and (dim-PT $)_{2}:(\mathrm{PCBM} @ b t)_{2 \mathrm{rw}}: e$ models. Photo-absorption cross sections for geometries randomly chosen from the MD simulations for the (dim-PT) $)_{2}(\mathrm{PCBM} @ \mathrm{t})_{1}: f,(\operatorname{dim}-\mathrm{PT})_{2}(\mathrm{PCBM} @)_{1}: e$, $(\operatorname{dim}-\mathrm{PT})_{3}(\mathrm{PCBM} @ \mathrm{t})_{1}: f$, and $(\operatorname{dim}-\mathrm{PT})_{3}(\mathrm{PCBM} @ \mathrm{t})_{1}: e$ models. Geometries of the interface 

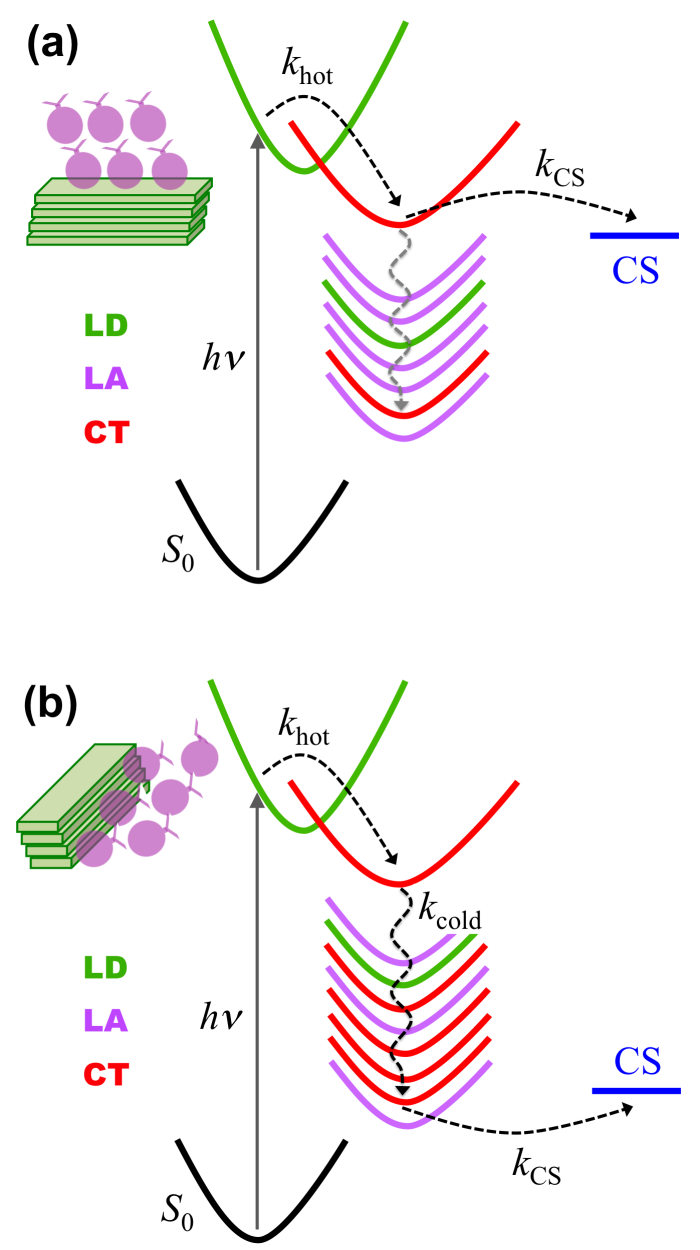

FIG. 12. Mechanisms for charge separation. (a) Face-on orientation. Photoexcitation to a LD bright state is followed by non-adibatic population transfer to a hot CT state (rate constant $k_{\text {hot }}$ ). Decay to the cold CT states (gray wavy line) is not likely to take place, so charge separation mostly proceeds from the hot CT exciton (rate constant $k_{\mathrm{CS}}$ ). (b) Edge-on orientation. Relaxation from the hot $\mathrm{CT}$ exciton to the cold $\mathrm{CT}$ excitons (rate constant $k_{\text {cold }}$ ) is favored by the high density of low-energy CT states. Charge separation mostly proceeds from the cold CT excitons at the bottom of the excitation band.

models and OB2 Slater-Koster parameters

\section{ACKNOWLEDGMENTS}

M. T. do N. V. acknowledges support from FAPESP (grant 2018/22948-0) and also from the Brazilian National Council for Scientific and Technological Development (CNPq), grant 304571/2018-0. The calculations were partly performed with HPC resources from STI (University of São Paulo), Centro Nacional de Processamento de Alto Desempenho em São Paulo 
(CENAPAD-SP), and the Mesocentre at the Aix Marseille University (project Equip@Meso, ANR-10-EQPX-29-01). V.Q.V. acknowledges support by an Energy Science and Engineering Fellowship of the Bredesen Center for Interdisciplinary Research and Graduate Education at the University of Tennessee, Knoxville. S.I. acknowledges support by the Laboratory Directed Research and Development (LDRD) Program of Oak Ridge National Laboratory. ORNL is managed by UT-Battelle, LLC, for DOE under contract DE-AC05-00OR22725. T.N. would like to thank the Laboratoire d'excellence iMUST for financial support. M. B. thanks the support of the Excellence Initiative of the Aix Marseille University ( $A^{*}$ MIDEX).

\section{REFERENCES}

${ }^{1}$ T. M. Clarke, and J. R. Durrant, Chem. Rev. 110, 6736 (2010).

${ }^{2}$ J. Benduhn, K. Tvingstedt, F. Piersimoni, S. Ullbrich, Y. Fan4, M. Tropiano, K. A. McGarry, O. Zeika, M. K. Riede, C. J. Douglas, S. Barlow, S. R. Marder, D. Neher, D. Spoltore, and K. Vandewal, Nat. Energy 7, 17053 (2017).

${ }^{3}$ K. Vandewal, Annu. Rev. Phys. Chem. 67, 113 (2016).

${ }^{4}$ V. Coropceanu, X-K. Chen, T. Wang, Z. Zheng, and J.-L. Brédas, Nat. Rev. Mater. 4, 689 (2019).

${ }^{5}$ S. D. Dimitrov, M. Azzouzi, J. Wu, J. Yao, Y. Dong, P. Shakya Tuladhar, B. C. Schroeder, E. R. Bittner, I. McCulloch, J. Nelson, and J. R. Durrant, J. Am. Chem. Soc. 141, 4634 (2019).

${ }^{6}$ T. R. Kafle, B. Kattel, S. Wanigasekara, T. Wang, and W.-L. Chan, Adv. Energy Mater. 10, 1904013 (2020).

${ }^{7}$ L. Tanguy, A. Fleury, P.-L. Karsenti, G. Brisard, A. Soldera, and P. D. Harvey, J. Phys. Chem. C 124, 16248 (2020).

${ }^{8}$ W. Zhao, S. Li, H. Yao, S. Zhang, Y. Zhang, B. Yang, and J. Hou, J. Am. Chem. Soc. 139, 7148 (2017).

${ }^{9}$ L. Meng, Y. Zhang, X. Wan, C. Li, X. Zhang, Y. Wang, X. Ke, Z. Xiao, L. Ding, R. Xia, H.-L. Yip,Y. Cao, and Y. Chen, Science 361, 1094 (2018).

${ }^{10}$ J. Yuan, Y. Zhang, L. Zhou, G. Zhang, H.-L. Yip, T.-K. Lau, X. Lu, C. Zhu, H. Peng, P. A. Johnson, M. Leclerc, Y. Cao, J. Ulanski, Y. Li, and Y. Zou, Joule 3, 1140 (2019). 
${ }^{11}$ Y. Cui, H. Yao, J. Zhang, T. Zhang, Y. Wang, L. Hong, K. Xian, B. Xu, S. Zhang, J. Peng, Z. Wei, F. Gao, and J. Hou Nat. Commun. 10, 2515 (2019).

${ }^{12}$ A. E. Jailaubekov, A. P. Willard, J. R. Tritsch, W. L. Chan, N. Sai, R. Gearba, L. G. Kaake, K. J. Williams, K. Leung, P. J. Rossky, X. Y. Zhu, Nat. Mater. 12, 662013.

${ }^{13}$ G. Grancini, M. Maiuri, D. Fazzi, A. Petrozza, H. J. Egelhaaf, D. Brida, G. Cerullo, G. Lanzani, Nat. Mater. 12, 29 (2013).

${ }^{14}$ B. M. Savoie, A. Rao, A. A. Bakulin, S. Gelinas, B. Movaghar, R. H. Friend, T. J. Marks, M. A. Ratner, J. Am. Chem. Soc. 136, 2876 (2014).

${ }^{15}$ T. G. J. van der Hofstad, D. Di Nuzzo, M. van den Berg, R. A. J. Janssen, S. C. J. Meskers, Adv. Energy Mater. 2, 1095 (2012).

${ }^{16}$ K. Vandewal, S. Albrecht, E. T. Hoke, K. R. Graham, J. Widmer, J. D. Douglas, M. Schubert, W. R. Mateker, J. T. Bloking, G. F. Burkhard, A. Sellinger, J. M. J. Frechet, A. Amassian, M. K. Riede, M. D. McGehee, D. Neher, A. Salleo, Nat. Mater. 13, 63 (2014). ${ }^{17}$ B. A. Gregg, J. Phys. Chem. Lett. 2, 3013 (2011).

${ }^{18}$ N. R. Monahan, K. W. Williams, B. Kumar, C. Nuckolls, and X.-Y. Zhu, Phys. Rev. Lett. 114, $247003(2015)$.

${ }^{19}$ Y. Yao, X. Xie, and H. Ma, . Phys. Chem. Lett. 7, 4830 (2016).

${ }^{20}$ R. S. Sanchez-Carrera, P. Paramonov, G. M. Day, V. Coropceanu, and J.-L. Brédas, J. Am. Chem. Soc. 132, 14437 (2010).

${ }^{21}$ S. M. Falke, C. A. Rozzi, D. Brida, M. Maiuri, M. Amato, E. Sommer, A. De Sio, A. Rubio, G. Cerullo, E. Molinari, and C. Lienau, Science 344, 1001 (2014)

${ }^{22}$ X.-K. Chen, V. Coropceanu, and J.-L. Brédas, Nat. Commun. 9, 5295 (2018).

${ }^{23}$ D. C.Coffey, B. W. Larson, A. W. Hains, J. B. Whitaker, J. B. N. Kopidakis, O. V. Boltalina, S. H. Strauss, and G. Rumbles, J. Phys. Chem. C 116, (2012).

${ }^{24}$ S. D. Dimitrov, A. A. Bakulin, C. B. Nielsen, B. C. Schroeder, J. Du, H. Bronstein, I. McCulloch, R. H. Friend, and J. R. Durrant, J. Am. Chem. Soc. 134, 18189 (2012).

${ }^{25}$ A. A. Bakulin, S. D. Dimitrov, A. Rao, P. C. Y. Chow, C. B. Nielsen, B. C. Schroeder, I. McCulloch, H. J. Bakker, J. R. Durrant, and R. H. Friend, J. Phys. Chem. Lett. 4, 209 (2013).

${ }^{26}$ K. Kawashima, Y. Tamai, H. Ohkita, I. Osaka, and K. Takimiya, Nat. Commun. 6, 10085 (2015). 
${ }^{27}$ J. Liu, S. Chen, D. Qian, B. Gautam, G. Yang, J. Zhao, J. Bergqvist, F. Zhang, W. Ma, H. Ade, and O. Inganäs, Nat. Energy 1, 1 (2016).

${ }^{28}$ P. Cheng, G. Li, X. W. Zhan, Y. Yang, Nat. Photonics 12, 131 (2018).

${ }^{29}$ S. Chen, Y. Wang, L. Zhang, J. Zhao, Y. Chen, D. Zhu, H. Yao, G. Zhang, W. Ma, R. H. Friend, and P. C. Chow, Adv. Mater. 30, 1804215 (2018).

${ }^{30}$ D. Qian, Z. Zheng, H. Yao, W. Tress, T. R. Hopper, S. Chen, S. Li, J. Liu, S. Chen, J. Zhang, and X. K. Liu, X.K., Nat. Mater. 17, 703 (2018).

${ }^{31}$ T. Saito, S.-I. Natsuda, K. Imakita, Y. Tamai, and H. Ohkita, Sol. RRL doi:10.1002/solr.202000255 (2020).

${ }^{32}$ N. R. Tummala, Z. Zheng, S. G. Aziz, V. Coropceanu, and J.-L. Brédas, J. Phys. Chem. Lett. 6, 3657 (2015).

${ }^{33}$ N. R. Tummala, S. A. Elroby, S. G. Aziz, C. Risko, V. Coropceanu, V. and J.-L. Brédas, J. Phys. Chem. C 120, 17242 (2016).

${ }^{34}$ Z. Zheng, N. R. Tummala, Y. T. Fu, V. Coropceanu, and J.-L. Brédas, ACS Appl. Mater. Inter. 9, 18095 (2017)

${ }^{35}$ L. Shi, C. K. Lee, and A. P. Willard, ACS Cent. Sci. 3, 1262 (2017).

${ }^{36}$ Z. Zheng, N. R. Tummala, T. Wang, V. Coropceanu, and J.-L. Brédas, Adv. Energy Mater. 9, 1803926 (2019).

${ }^{37}$ H. Jiang, P. Hu, J. Ye, K. K. Zhang, Y. Long, W. Hu and C. Kloc, J. Mater. Chem. C, 6 1884 (2018).

${ }^{38}$ G. Han, and Y. Yi, J. Phys. Chem Lett. 102911 (2019).

${ }^{39}$ Y. Xu, H. Yao, L. Ma, L. Hong, J. Li, Q. Liao, Y. Zu, J. Wang, M. Gao, L. Ye, and J. Hou, Angew. Chem. Int. Ed. 132, 9089 (2020).

${ }^{40}$ H. Tamura and I. Burghardt, J. Am. Chem. Soc. 135, 16364 (2013).

${ }^{41}$ S. Gélinas, A. Rao, A. Kumar, S. L. Smith, A. W. Chin, J. Clark, T. S. van der Poll, G. C. Bazan, R. H. Friend, Science 343, 512 (2014).

${ }^{42}$ B. Yang, Y. Yi, C. R. Zhang, S. G. Aziz, V. Coropceanu, and J.-L. Brédas, J. Phys. Chem. C 118, 27648 (2014).

${ }^{43}$ B. Bernardo, D. Cheyns, B. Verreet, R. D. Schaller, B. P. Rand, N. C. Giebink, Nat. Commun. 5, 3245 (2014).

${ }^{44}$ G. D'Avino, L. Muccioli, Y. Olivier, and D. Beljonne, J. Phys. Chem. Lett. 7, 536 (2016). 
${ }^{45}$ V. Abramavicius, V. Pranculis, A. Melianas, O. Inganäs, V. Gulbinas, and D. Abramavicius, Sci. Rep. 6, 32914 (2016).

${ }^{46}$ X. Shen, G. Han, and Y. Yi, Phys. Chem. Chem. Phys. 18, 15955 (2016).

${ }^{47}$ X.-K. Chen, M. K. Ravva, H. Li, S. M. Ryno, J.-L. Brédas, Adv. Energy Mater. 6, 1601325 (2016).

${ }^{48}$ C. Sutton, N. R. Tummala, T. Kemper, S. G. Aziz, J. Sears, V. Coropceanu, and J.-L. Brédas, J. Chem. Phys. 146, 224705 (2017).

${ }^{49}$ A. Gluchowski, K. L. G. Gray, S. N. Hood, and I. Kassal, J. Phys. Chem. Lett. 9, 1359 (2018).

${ }^{50}$ N. Felekidis, A. Melianas, and M. Kemerink, J. Phys. Chem. Lett. 11, 3563 (2020).

${ }^{51}$ N. A. Ran, S. Roland, J. A. Love, V. Savikhin, C. J. Takacs, Y.-T. Fu, H. Li, V. Coropceanu, X. Liu, J.-L. Brédas, G. C. Bazan, M. F. Toney, D. Neher, and T.-Q. Nguyen, Nat. Commun. 8, 79 (2017).

${ }^{52}$ D. Fazzi, M. Barbatti, and W. Thiel, J. Phys. Chem. Lett. 8, 4727 (2017).

${ }^{53}$ Y. L. Lin, M. A. Fusella, and B. P. Rand, Adv. Energy Mater. 8, 1702816 (2018).

${ }^{54}$ G. Han, Y. Yi, and Z. Shuai, Adv. Energy Mater. 8, 1702743 (2018).

${ }^{55}$ T.A. Niehaus, J. Mol. Struct.: THEOCHEM 914, 38 (2009).

${ }^{56}$ A. Humeniuk, and R. Mitrić, J. Chem. Phys. 143, 134120 (2015).

${ }^{57}$ V. Lutsker, B. Aradi, and T. A. Niehaus, J. Chem. Phys. 143, 184107 (2015).

${ }^{58}$ J. J. Kranz, M. Elstner, B. Aradi, T. Frauenheim, V. Lutsker, A. D. Garcia, and T. A. Niehaus, J. Chem. Theory Comput. 13, 1737 (2017).

${ }^{59}$ F. Plasser, and H. Lischka, J. Chem. Theory Comput. 8, 2777 (2012).

${ }^{60}$ A. Mewes, J.-M. Mewes, F. Plasser, and A. Dreuw, Phys. Chem. Chem. Phys. 18, 2548 (2016).

${ }^{61}$ F. Plasser, J. Chem. Phys. 152, 084108 (2020).

${ }^{62}$ S. Hedström, Q. Tao, E. Wang, P. Persson, Phys. Chem. Chem. Phys. 17, 26677 (2015).

${ }^{63}$ Q. Tao, Y. Xia, X. Xu, S. Hedström, O. Bäcke, D. I. James, P. Persson, E. Olsson, O. Inganäs, L. Hou, and W. Zhu, Macromolecules 48, 1009 (2015).

${ }^{64}$ M. L. Keshtov, A. R. Khokhlov, S. A. Kuklin, F. C. Chen, E. N. Koukaras, and G. D. Sharma, ACS Appl. Mater. Inter. 8, 32998 (2016).

${ }^{65}$ Y. Wang, S. W. Kim, J. Lee, H. Matsumoto, B. J. Kim, and T. Michinobu, ACS Appl. Mater. Inter. 11, 22583 (2019). 
${ }^{66}$ P. Li, L. Xu, H. Shen, X. Duan, J. Zhang, Z. Wei, Z. Yi, C. A. Di, and S. Wang, ACS Appl. Mater. Inter. 88620 (2016).

${ }^{67}$ Y. Wang, T. Hasegawa, H. Matsumoto, T. Mori, T. and T. Michinobu, Adv. Funct. Mater. 27, 1701486 (2017).

${ }^{68}$ L. Chen, P. Yin, X. Zeng, C. Weng, Y. Chen, C. Cui, and P. Shen, Polymer, 182, 121850 (2019).

${ }^{69}$ B. Aradi, B. Hourahine, and T. Frauenheim, . J. Phys. Chem. A 111, 5678 (2007).

${ }^{70}$ M. Elstner, D. Porezag, G. Jungnickel, J. Elsner, M. Haugk, T. Frauenheim, S. Suhai, and G. Seifert, Phys. Rev. B 58, 7260 (1998).

${ }^{71}$ R. Baer, and D. Neuhauser, Phys. Rev. Lett. 94, 043002 (2005).

${ }^{72}$ E. Livshits, and R. Baer, Phys. Chem. Chem. Phys. 9293 (2007).

${ }^{73}$ V. Q. Vuong, J. A. Kuriappan, M. Kubillus, J. J. Kranz, T. Mast, T. A. Niehaus, S. Irle, and M. Elstner, J. Chem. Theory Comput. 14, 115 (2018).

${ }^{74}$ M. Elstner, P. Hobza, T. Frauenheim, S. Suhai, and E. Kaxiras, J. Chem. Phys. 114, 5149 (2001).

${ }^{75}$ L. Stojanović, S. G. Aziz, R. H. Hilal, F. Plasser, T. A. Niehaus, and M. Barbatti, J. Chem. Theory Comput. 13, 5846 (2017).

${ }^{76}$ F. Plasser, THEODORE: A package for theoretical density, orbital relaxation, and exciton analysis, 2019, available at http://theodore-qc.sourceforge.net.

${ }^{77}$ M. J. Frisch, G. W. Trucks, H. B. Schlegel, G. E. Scuseria, M. A Robb, J. R. Cheeseman, G. Scalmani, V. Barone, B. Mennucci, G. A. Petersson et al., Gaussian 09, revision D.01, Gaussian, Inc., Wallingford, CT, 2009.

${ }^{78}$ R. Crespo-Otero and M. Barbatti, Theor. Chem. Acc. 131, 1237 (2012).

${ }^{79}$ M. Barbatti, M. Ruckenbauer, F. Plasser, J. Pittner, G. Granucci, M. Persico, and H. Lischka, Comp. Mol. Sci., 4, 26 (2014).

${ }^{80}$ M. Barbatti, G. Granucci, M. Ruckenbauer, F. Plasser, R. Crespo-Otero, J. Pittner, M. Persico, and H. Lischka, NEWTON-X: A package for Newtonian Dynamics Close to the Crossing Seam (v. 2.2). Available via the Internet at www.newtonx.org, 2018.

${ }^{81}$ A. Darghouth, M. Casida, W. Taouali, K. Alimi, M. Ljungberg, P. Koval, D. SánchezPortal, and D. Foerster, Computation 3, 616 (2015).

${ }^{82}$ A. A. M. H. M. Darghouth, G. C. Correa, S. Juillard, M. E. Casida, A. Humeniuk, and R. Mitrić, J. Chem. Phys. 149, 134111 (2018). 
${ }^{83}$ A. A. H. M. Darghouth, M. E. Casida, X. Zhu, B. Natarajan, H. Su, A. Humeniuk, E. Titov, X. Miao, R. Mitric, arXiv 1803.11121 (2020). 
TABLE II. Lowest-lying absorption bands of PTBTBTz:PCBM models. In each case, we show the major contributions from the vertical excited states of the stationary structures, indicating the energies, in $\mathrm{eV}$, and the oscillator strengths (given in parenthesis). The letter in parenthesis indicates the interface in Fig. 3. The two interfaces with $\left(^{*}\right)$ are shown in Fig. S3 a and b of the Supporting Information.

\begin{tabular}{|c|c|c|c|}
\hline System & $1^{\text {st }}$ band & $2^{\text {nd }}$ band & $3^{\text {rd }}$ band \\
\hline$(\operatorname{dim}-\mathrm{PT})_{2}(\mathrm{PCMB} @ \mathrm{bt})_{1}: f(\mathrm{a})$ & $2.557(2.245)$ & $2.778(1.345)$ & weak \\
\hline \multirow{2}{*}{$(\operatorname{dim}-\mathrm{PT})_{2}(\mathrm{PCMB} @ b t z)_{1}: f(*)$} & $2.534(0.919)$ & $2.802(0.808)$ & weak \\
\hline & $2.547(1.177)$ & $2.806(0.609)$ & \\
\hline$(\operatorname{dim}-\mathrm{PT})_{2}(\mathrm{PCMB} @ \mathrm{t})_{1}: f(\mathrm{~b})$ & $2.612(2.572)$ & $2.775(0.919)$ & weak \\
\hline \multirow[t]{2}{*}{$(\operatorname{dim}-\mathrm{PT})_{2}(\mathrm{PCMB} @ b t)_{1}: e(\mathrm{c})$} & $2.518(0.183)$ & $2.771(1.266)$ & $3.142(0.737)$ \\
\hline & $2.587(2.239)$ & & \\
\hline \multirow[t]{2}{*}{$(\operatorname{dim}-\mathrm{PT})_{2}(\mathrm{PCMB} @ \mathrm{t})_{1}: e(\mathrm{~d})$} & $2.613(2.406)$ & $2.709(0.923)$ & $3.071(0.228)$ \\
\hline & $2.780(0.576)$ & & $3.143(0.561)$ \\
\hline \multirow[t]{3}{*}{$(\operatorname{dim}-\mathrm{PT})_{3}(\mathrm{PCMB} @ \mathrm{bt})_{1}: f(\mathrm{e})$} & $2.465(1.558)$ & $2.691(0.591)$ & $2.940(0.990)$ \\
\hline & & $2.696(1.231)$ & $2.945(0.176)$ \\
\hline & & $2.706(0.423)$ & \\
\hline \multirow[t]{2}{*}{$(\operatorname{dim}-\mathrm{PT})_{3}(\mathrm{PCMB} @ b t z)_{1}: f(*)$} & $2.460(1.512)$ & $2.702(2.197)$ & $2.934(1.237)$ \\
\hline & & & $2.828(0.215)$ \\
\hline \multirow{2}{*}{$(\operatorname{dim}-\mathrm{PT})_{3}(\mathrm{PCMB} @ \mathrm{t})_{1}: f(\mathrm{f})$} & $2.428(0.149)$ & $2.636(0.516)$ & $2.937(1.061)$ \\
\hline & $2.471(1.319)$ & $2.710(2.171)$ & \\
\hline \multirow[t]{2}{*}{$(\operatorname{dim}-\mathrm{PT})_{3}(\mathrm{PCMB} @ \mathrm{bt})_{1}: e(\mathrm{~g})$} & $2.464(1.572)$ & $2.723(2.453)$ & $2.969(1.165)$ \\
\hline & & & $3.036(0.130)$ \\
\hline \multirow[t]{3}{*}{$(\operatorname{dim}-\mathrm{PT})_{3}(\mathrm{PCMB} @ \mathrm{t})_{1}: e(\mathrm{~h})$} & $2.490(0.860)$ & $2.603(0.149)$ & $2.914(0.174)$ \\
\hline & & $2.678(3.244)$ & $2.917(0.776)$ \\
\hline & & & $2.931(0.120)$ \\
\hline \multirow[t]{3}{*}{$(\operatorname{dim}-\mathrm{PT})_{2}\left(\mathrm{PCMB} @ b t \_b t z\right)_{2 s d}: f(\mathrm{i})$} & $2.526(2.040)$ & $2.790(1.110)$ & $2.880(0.108)$ \\
\hline & & $2.804(0.159)$ & $2.887(0.138)$ \\
\hline & & & $2.943(0.147)$ \\
\hline \multirow[t]{2}{*}{$(\operatorname{dim}-\mathrm{PT})_{2}\left(\mathrm{PCMB} @ \mathrm{t} \_\mathrm{t}\right)_{2 s d}: f(\mathrm{j})$} & $2.531(2.032)$ & $2.807(1.036)$ & $2.941(0.211)$ \\
\hline & & $2.869(0.269)$ & $3.070(0.111)$ \\
\hline \multirow[t]{3}{*}{$(\operatorname{dim}-\mathrm{PT})_{2}\left(\mathrm{PCMB} @ \mathrm{t} \_\mathrm{t}\right)_{2 s d}: e(\mathrm{~m})$} & $2.632(0.461)$ & $2.731(0.822)$ & weak \\
\hline & $2.636(0.877)$ & & \\
\hline & $2.652(1.606)$ & & \\
\hline \multirow[t]{3}{*}{$(\operatorname{dim}-\mathrm{PT})_{2}(\mathrm{PCMB} @ \mathrm{bt})_{2 r w}: f(\mathrm{k})$} & $2.571(2.236)$ & $2.793(1.008)$ & $2.900(0.129)$ \\
\hline & & $2.799(0.114)$ & $2.955(0.111)$ \\
\hline & & & $3.173(0.592)$ \\
\hline \multirow[t]{2}{*}{$(\operatorname{dim}-\mathrm{PT})_{2}(\mathrm{PCMB} @ \mathrm{t})_{2 r w}: f(\mathrm{l})$} & $2.569(2.035)$ & $2.713(0.207)$ & $2.900(0.129)$ \\
\hline & $2.602(0.185)$ & $2.787(1.110)$ & $3.173(0.600)$ \\
\hline \multirow[t]{3}{*}{$(\operatorname{dim}-\mathrm{PT})_{2}(\mathrm{PCMB} @ \mathrm{bt})_{2 r w}: e(\mathrm{n})$} & $2.579(2.316)$ & $2.754(0.235)$ & $2.915(0.388)$ \\
\hline & & $2.794(0.880)$ & $2.964(0.231)$ \\
\hline & & & $3.172(0.552)$ \\
\hline \multirow[t]{2}{*}{$(\operatorname{dim}-\mathrm{PT})_{2}(\mathrm{PCMB} @ \mathrm{t})_{2 r w}: e(\mathrm{o})$} & $2.628(2.481)$ & $2.723(0.422)$ & $2.921(0.145)$ \\
\hline & & $2.773(0.861)$ & $3.159(1.081)$ \\
\hline
\end{tabular}


TABLE III. Average number of cold CT excitons defined with respect to the lowest-lying CT state, $\left\langle n_{\mathrm{CT}}^{\text {cold }}\right\rangle$, and with respect to the $S_{1}$ state, $\left\langle n_{\mathrm{CT}}^{\text {cold }}\right\rangle$ (see text). The average number of CT states lying below the brightest state, $\left\langle n_{\mathrm{CT}}^{\text {low }}\right\rangle$, and the average values of the charge-transfer number for the cold CT excitons defined with respect to $S_{1},\left\langle\mathrm{CT}_{\text {cold }}\right\rangle$, are also shown. For each system, the averages are computed for the different relative positions of the PCBM molecules (bt, btz, $\mathrm{t}$ ) and also without discriminating the positions (all). The number of geometries, $N$, used to calculate the $n_{\mathrm{CT}}$ averages and standard deviations (given in parenthesis) is indicated. The $\mathrm{CT}_{\text {cold }}$ values are averaged over the CT states, not over the geometries. The letter in parenthesis indicates the interface in Fig. 3. The two interfaces with $\left(^{*}\right)$ are shown in Fig. S3 a and b of the Supporting Information.

\begin{tabular}{|c|c|c|c|c|c|}
\hline System & $N$ & $\left\langle n_{\mathrm{CT}}^{\text {low }}\right\rangle$ & $\left\langle n_{\mathrm{CT}}^{\text {cold } \star}\right\rangle$ & $\left\langle n_{\mathrm{CT}}^{\text {cold }}\right\rangle$ & $\left\langle\mathrm{CT}_{\text {cold }}\right\rangle$ \\
\hline$(\operatorname{dim}-\mathrm{PT})_{2}(\mathrm{PCMB} @ \mathrm{bt})_{1}: f(\mathrm{a})$ & 10 & $(0.9 \pm 0.5)$ & $(0.8 \pm 0.4)$ & $(0.1 \pm 0.3)$ & $(0.83 \pm 0.00)$ \\
\hline$(\operatorname{dim}-\mathrm{PT})_{2}(\mathrm{PCMB} @ \mathrm{btz})_{1}: f(*)$ & 10 & $(2.5 \pm 0.7)$ & $(1.9 \pm 0.3)$ & $(1.4 \pm 0.5)$ & $(0.87 \pm 0.05)$ \\
\hline$(\operatorname{dim}-\mathrm{PT})_{2}(\mathrm{PCMB} @ \mathrm{t})_{1}: f(\mathrm{~b})$ & 10 & $(2.7 \pm 0.5)$ & $(1.9 \pm 0.3)$ & $(1.7 \pm 0.6)$ & $(0.84 \pm 0.03)$ \\
\hline$(\operatorname{dim}-\mathrm{PT})_{2}(\mathrm{PCMB} @ \text { all })_{1}: f$ & 30 & $(2.0 \pm 1.0)$ & $(1.5 \pm 0.6)$ & $(1.1 \pm 0.9)$ & $(0.86 \pm 0.05)$ \\
\hline$(\operatorname{dim}-\mathrm{PT})_{2}(\mathrm{PCMB} @ b t)_{1}: e(\mathrm{c})$ & 10 & $(4.4 \pm 0.8)$ & $(2.8 \pm 0.7)$ & $(2.2 \pm 0.7)$ & $(0.96 \pm 0.05)$ \\
\hline$(\operatorname{dim}-\mathrm{PT})_{2}(\mathrm{PCMB} @ \mathrm{t})_{1}: e(\mathrm{~d})$ & 10 & $(5.3 \pm 0.6)$ & $(3.7 \pm 1.2)$ & $(2.8 \pm 1.6)$ & $(0.99 \pm 0.02)$ \\
\hline$(\operatorname{dim}-\mathrm{PT})_{2}(\mathrm{PCMB} @ \text { all })_{1}: e$ & 20 & $(4.9 \pm 0.9)$ & $(3.3 \pm 1.1)$ & $(2.5 \pm 1.3)$ & $(0.97 \pm 0.04)$ \\
\hline$(\operatorname{dim}-\mathrm{PT})_{3}(\mathrm{PCMB} @ \mathrm{bt})_{1}: f(\mathrm{e})$ & 5 & $(1.8 \pm 1.2)$ & $(1.4 \pm 0.5)$ & $(0.6 \pm 0.5)$ & $(0.86 \pm 0.03)$ \\
\hline$(\operatorname{dim}-\mathrm{PT})_{3}(\mathrm{PCMB} @ \mathrm{btz})_{1}: f\left(^{*}\right)$ & 5 & $(2.2 \pm 1.2)$ & $(1.4 \pm 0.5)$ & $(1.2 \pm 0.7)$ & $(0.93 \pm 0.02)$ \\
\hline$(\operatorname{dim}-\mathrm{PT})_{3}(\mathrm{PCMB} @ \mathrm{t})_{1}: f(\mathrm{f})$ & 5 & $(1.8 \pm 1.0)$ & $(1.2 \pm 0.4)$ & $(1.2 \pm 0.4)$ & $(0.87 \pm 0.06)$ \\
\hline$(\operatorname{dim}-\mathrm{PT})_{3}(\mathrm{PCMB} @ \text { all })_{1}: f$ & 15 & $(1.9 \pm 1.1)$ & $(1.3 \pm 0.5)$ & $(1.0 \pm 0.6)$ & $(0.89 \pm 0.05)$ \\
\hline$(\operatorname{dim}-\mathrm{PT})_{3}(\mathrm{PCMB} @ \mathrm{bt})_{1}: e(\mathrm{~g})$ & 5 & $(6.4 \pm 2.1)$ & $(2.0 \pm 0.6)$ & $(2.0 \pm 0.6)$ & $(0.99 \pm 0.01)$ \\
\hline$(\operatorname{dim}-\mathrm{PT})_{3}(\mathrm{PCMB} @ \mathrm{t})_{1}: e(\mathrm{~h})$ & 5 & $(9.6 \pm 1.0)$ & $(3.2 \pm 1.5)$ & $(3.2 \pm 1.5)$ & $(0.998 \pm 0.002)$ \\
\hline$(\operatorname{dim}-\mathrm{PT})_{3}(\mathrm{PCMB} @ \text { all })_{1}: e$ & 10 & $(8.0 \pm 2.2)$ & $(2.6 \pm 1.3)$ & $(2.6 \pm 1.3)$ & $(0.995 \pm 0.009)$ \\
\hline$(\operatorname{dim}-\mathrm{PT})_{2}\left(\mathrm{PCMB} @ b t \_b t z\right)_{2 s d}: f(\mathrm{i})$ & 5 & $(3.6 \pm 1.7)$ & $(1.8 \pm 0.7)$ & $(1.4 \pm 0.5)$ & $(0.88 \pm 0.08)$ \\
\hline$(\operatorname{dim}-\mathrm{PT})_{2}\left(\mathrm{PCMB} @ \mathrm{t} \_\mathrm{t}\right)_{2 s d}: f(\mathrm{j})$ & 5 & $(1.8 \pm 1.7)$ & $(1.4 \pm 1.0)$ & $(0.8 \pm 1.2)$ & $(0.85 \pm 0.06)$ \\
\hline$(\operatorname{dim}-\mathrm{PT})_{2}(\mathrm{PCMB} @ \text { all })_{2 s d}: f$ & 10 & $(2.7 \pm 1.9)$ & $(1.6 \pm 0.9)$ & $(1.1 \pm 0.9)$ & $(0.88 \pm 0.07)$ \\
\hline$(\operatorname{dim}-\mathrm{PT})_{2}\left(\mathrm{PCMB} @ \mathrm{t} \_\mathrm{t}\right)_{2 s d}: e(\mathrm{~m})$ & 5 & $(10.2 \pm 0.8)$ & $(4.8 \pm 1.7)$ & $(4.0 \pm 1.7)$ & $(0.96 \pm 0.03)$ \\
\hline$(\operatorname{dim}-\mathrm{PT})_{2}(\mathrm{PCMB} @ \mathrm{bt})_{2 r w}: f(\mathrm{k})$ & 5 & $(1.5 \pm 1.1)$ & $(1.3 \pm 0.8)$ & $(0.8 \pm 0.8)$ & $(0.93 \pm 0.04)$ \\
\hline$(\operatorname{dim}-\mathrm{PT})_{2}(\mathrm{PCMB} @ \mathrm{t})_{2 r w}: f(\mathrm{l})$ & 5 & $(1.0 \pm 0.7)$ & $(1.0 \pm 0.7)$ & $(1.0 \pm 0.7)$ & $(0.88 \pm 0.04)$ \\
\hline$(\operatorname{dim}-\mathrm{PT})_{2}(\mathrm{PCMB} @ \text { all })_{2 r w}: f$ & 10 & $(1.3 \pm 0.9)$ & $(1.2 \pm 0.7)$ & $(0.8 \pm 0.7)$ & $(0.91 \pm 0.04)$ \\
\hline$(\operatorname{dim}-\mathrm{PT})_{2}(\mathrm{PCMB} @ b t)_{2 r w}: e(\mathrm{n})$ & 5 & $(2.6 \pm 0.5)$ & $(2.4 \pm 0.8)$ & $(0.2 \pm 0.4)$ & $(0.89 \pm 0.00)$ \\
\hline$(\operatorname{dim}-\mathrm{PT})_{2}(\mathrm{PCMB} @ \mathrm{t})_{2 r w}: e(\mathrm{o})$ & 5 & $(4.4 \pm 0.5)$ & $(2.8 \pm 1.0)$ & $(1.2 \pm 1.2)$ & $(0.98 \pm 0.02)$ \\
\hline$(\operatorname{dim}-\mathrm{PT})_{2}(\mathrm{PCMB} @ \text { all })_{2 r w}: e$ & 10 & $(3.5 \pm 1.0)$ & $(2.6 \pm 0.9)$ & $(0.7 \pm 1.0)$ & $(0.97 \pm 0.04)$ \\
\hline
\end{tabular}

